Draft Version OCtober 17, 2018

Preprint typeset using $\mathrm{LATE}_{\mathrm{E}} \mathrm{X}$ style emulateapj v. 04/17/13

\title{
PLANETARY POPULATION SYNTHESIS COUPLED WITH ATMOSPHERIC ESCAPE: A STATISTICAL VIEW OF EVAPORATION
}

\author{
Sheng Jin ${ }^{1,2,3}$, Christoph Mordasini ${ }^{\star 2}$, Vivien Parmentier ${ }^{4}$, \\ Roy van Boekel ${ }^{2}$, Thomas Henning ${ }^{2}$, Jianghui Ji ${ }^{1,5}$ \\ 1 Purple Mountain Observatory, Chinese Academy of Sciences, Nanjing 210008, China; shengjin@pmo.ac.cn \\ 2 Max-Planck-Institut für Astronomie, Königstuhl 17, D-69117 Heidelberg, Germany; mordasini@mpia.de \\ ${ }^{3}$ University of Chinese Academy of Sciences, Beijing 100049, China \\ ${ }^{4}$ Laboratoire Lagrange, UMR7293, Université de Nice Sophia-Antipolis, CNRS, Observatoire de la Côte d'Azur, 06300 Nice, France and \\ ${ }^{5}$ Key Laboratory of Planetary Sciences, Chinese Academy of Sciences, Nanjing 210008, China; jijh@pmo.ac.cn \\ Draft version October 17, 2018
}

\begin{abstract}
We apply hydrodynamic evaporation models to different synthetic planet populations that were obtained from a planet formation code based on a core-accretion paradigm. We investigated the evolution of the planet populations using several evaporation models, which are distinguished by the driving force of the escape flow (X-ray or EUV), the heating efficiency in energy-limited evaporation regimes, or both. Although the mass distribution of the planet populations is barely affected by evaporation, the radius distribution clearly shows a break at approximately $2 R_{\oplus}$. We find that evaporation can lead to a bimodal distribution of planetary sizes (Owen \& Wu 2013) and to an "evaporation valley" running diagonally downwards in the orbital distance - planetary radius plane, separating bare cores from low-mass planet that have kept some primordial $\mathrm{H} / \mathrm{He}$. Furthermore, this bimodal distribution is related to the initial characteristics of the planetary populations because lowmass planetary cores can only accrete small primordial $\mathrm{H} / \mathrm{He}$ envelopes and their envelope masses are proportional to their core masses. We also find that the population-wide effect of evaporation is not sensitive to the heating efficiency of energy-limited description. However, in two extreme cases, namely without evaporation or with a $100 \%$ heating efficiency in an evaporation model, the final size distributions show significant differences; these two scenarios can be ruled out from the size distribution of Kepler candidates.
\end{abstract}

Subject headings: planets and satellites: atmospheres — planets and satellites: physical evolution planets and satellites: interiors

\section{INTRODUCTION}

The large number of exoplanets detected thus far provides significant observational constraints for theoretical studies on planet formation and evolution. The population-wide distributions of these exoplanets are closely related to their formation history. Based on the core accretion paradigm (Lin et al. 1996; Weidenschilling \& Marzari 1996; Ida \& Lin 2004; Mordasini et al. 2012a), we can generate synthetic planet populations that may explain the statistical characteristics of exoplanets, such as the planets semi-major axis versus its mass $(a-M)$ (Ida \& Lin 2004; Mordasini et al. 2009) and its mass-radius diagram (Mordasini et al. 2012b).

Recently, the Kepler mission detected thousands of planetary candidates, the radii of which were measured using transit observations (Borucki et al. 2011). These data may provide an important clue to the size distribution of close-in planets (Howard et al. 2012; Dong \& Zhu 2013; Petigura et al. 2013a), which is an essential test of the current planet formation theories. Previous planetary population synthesis models by Mordasini et al. (2012a b) reproduced a synthetic planet population with a similar planet size distribution as observed in the Kepler data at radii $>2 R_{\oplus}$. However, for planets with radii $<2 R_{\oplus}$, a strong decrease in the synthetic planet

${ }^{\star}$ Reimar-Lüst Fellow of the MPG population is inconsistent with the plateau in the planet size distribution of the Kepler data, after correction for observational bias (Howard et al. 2012; Dong \& Zhu 2013; Petigura et al. 2013a). The divergence may be due to simplifications in the planetary population synthesis models (Mordasini et al. 2012a, b). As a simplification, atmospheric evaporation after the protoplanetary disks have dissipated (Lammer et al. 2003; Baraffe et al. 2004; Murray-Clay et al. 2009), which is important for close-in planets, was not considered in the former studies. Nevertheless, atmospheric evaporation may play a vital role in the thermal evolution of low-mass planets; this can alter the size distribution of close-in planets with radii $<2 R_{\oplus}$ (Owen \& Jackson 2012; Lopez et al. 2012; Lammer et al. 2014). Thus in order to use the radius distribution of close-in exoplanets to constrain planet formation models which is our final goal, it is necessary to include the effect of atmospheric escape.

In the planetary evolution stage after disk dissipation, the atmospheric structure and thermal evolution of a close-in planet are strongly influenced by intense irradiation from the host star (Guillot \& Showman 2002; Fortney et al. 2005, 2008; Hansen 2008; Guillot 2010). Moreover, the incident stellar X-ray and extremeultraviolet (EUV) flux can drive hydrodynamic evaporation in the atmosphere of close-in planets and yield a substantially higher mass-loss rate than for planets in the Jeans escape regime (Lammer et al. 2003; Baraffe et al. 
2004; Murrav-Clay et al. 2009; Owen \& Jackson 2012; Lopez et al. 2012; Lammer et al. 2014). For example, oxygen, carbon, and magnesium were detected in the upper atmosphere of HD 209458b at a distance of several planetary radii, which indicates that its atmosphere is undergoing hydrodynamic escape (Vidal-Madjar et al. 2004, 2013). The estimated atmosphere mass-loss rate for HD 209458b is $\gtrsim 10^{10} \mathrm{~g} \mathrm{~s}^{-1}$ (Vidal-Madiar et al. 2004, 2008). Wu \& Lithwick (2013) calculated the masses of 22 sub-Jovian Kepler planet pairs with the orbital periods of $\sim 8$ days using the TTV data and found that the mass-radius relationship of these planets corresponds to the constant escape velocity $\sim 20 \mathrm{~km} \mathrm{~s}^{-1}$, which is similar to the sound speed of hydrogen plasma at $10^{4} \mathrm{~K}$, indicating that hydrodynamic evaporation is likely.

The atmospheric mass loss due to evaporation during planetary evolution can be estimated by considering an energy-limited model. This scenario assumes that a portion of the heating energy of stellar irradiation contributes to $P \mathrm{~d} V$ work that expands the upper atmosphere (Watson et al. 1981). The mass-loss rate in the energy-limited model depends on the strength of stellar XUV irradiation, including both X-ray and EUV flux, and the heating efficiency, which describes how much heating is converted to $P \mathrm{~d} V$ work. Following the temporal evolution of the XUV emission from a sun-like star and assuming $100 \%$ heating efficiency, early studies showed that even a Jovian planet can lose its entire initial envelope during its lifetime at close-in orbits (Lammer et al. 2003; Baraffe et al. 2004). However, later hydrodynamic simulations of EUV-driven atmospheric escape show that although the EUV-driven mass flow can produce the observed Lyman- $\alpha$ absorption signatures, this mechanism can only evaporate a small portion of the mass of a Jovian planet (Tian et al. 2005; Yelle 2004; Murrav-Clav et al. 2009; Owen \& Jackson 2012). Furthermore, Tian et al. (2005) showed that, in the energylimited model, the assumption that the incoming irradiation is absorbed inside a single layer is inaccurate because the absorption depth of the incident radiation depends on the wavelength. Murray-Clay et al. (2009) found that atmospheric evaporation is in a radiation-recombinationlimited regime for high EUV flux. In this regime, a large portion of the heating energy is lost to cooling radiation, which decreases the mass-loss rate. Owen \& Jackson (2012) showed that atmospheric evaporation can be either X-ray- or EUV-driven, depending on the X-ray and EUV flux. Most planets are in an X-ray-driven evaporation regime at the beginning of evolution and then transition to an EUV-driven regime when the X-ray flux falls below a critical value. This transition indicates that using the total XUV fluxes in an evaporation model is insufficient because, in various regimes, the escape flow is dominated by the stellar irradiation at different wavelengths, either X-ray or EUV (Owen \& Jackson 2012).

Unlike Jovian planets, Neptune-like planets and superEarths are most likely to lose their entire envelopes at small distances (Lammer et al. 2009; Lopez et al. 2012; Owen \& Jackson 2012). Because the mass-loss timescale for planets scales with the planetary mass $\times$ the planetary mean density, the planetary thermal evolution coupled with atmospheric escape can elucidate the observed threshold for low-density planets in the $M_{\mathrm{p}}^{2} / R_{\mathrm{p}}^{3}$ versus distance ${ }^{2}$ parameter space, above which there is no low-mass, low-density transiting exoplanets have not been found (Jackson et al. 2012; Lopez et al. 2012; Owen \& Wu 2013). Moreover, Owen \& Wu (2013) found that evaporation can create a bimodal planet size distribution around $2 R_{\oplus}$. In an extensive investigation of evaporation that included thousands of planets with different sizes and incident fluxes, Lopez \& Fortney (2013) recently showed that the bimodal distribution near $2 R_{\oplus}$ was unclear. In addition, they observed a diagonal region in the semi-major axis versus radius $(a-R)$ space, where planets are relatively rare.

Herein, we simulate the thermal evolution of synthetic planet populations that were obtained from a planet formation code (Alibert et al. 2005; Mordasini et al. $2012 \mathrm{a}, \mathrm{b}$ ). The atmospheric mass-loss due to hydrodynamic evaporation is now included in the planetary evolution. Thereby, we aim to determine how evaporation statistically affects the entire planet population and to make a more consistent comparison with the Kepler data. Our results show that the $a-R$ space is strongly influenced by evaporation, which can modify the size distribution of the planets within $1 \mathrm{AU}$.

The paper is organized as follows. In $\S 2$ we describe the evaporation models used herein. Our improved atmospheric model and numerical experiments on planet evolution are shown in $\S$. In $\S$, we present the mass and radius distributions of the synthetic planet populations and compare the planet size distribution of the synthetic populations with Kepler candidates. Finally, we provide a detailed discussion $(\S 5$ and brief summary $(\S$ 6).

\section{EVAPORATION MODELS}

The dominant heating source of hydrodynamic outflow is either EUV or X-ray radiation, which divides the hydrodynamic evaporation into two distinct sub-regimes (Owen \& Jackson 2012). In this work, we use a model that includes both EUV-driven and X-ray-driven evaporation through simple semi-analytical equations.

\subsection{X-ray-driven Evaporation}

$\mathrm{X}$-ray photons have a smaller interaction cross-section compared with EUV photons; Thus, they penetrate deeper into the planetary atmosphere. In the early stage of planetary evolution, X-ray irradiation is an important heating source for planetary atmospheres due to strong X-ray emissions from young stars (Ribas et al. 2005; Jackson et al. 2012). In this work, the mass-loss rate in an X-ray-driven regime is calculated using an an energy-limited model and by assuming that part of the heating energy is converted to $P \mathrm{~d} V$ work with the efficiency factor $\epsilon$ (Jackson et al. 2012):

$$
\dot{m}=\epsilon \frac{16 \pi F R_{\mathrm{p}}^{3}}{3 G M_{\mathrm{p}} K(\xi)}
$$

where $M_{\mathrm{p}}$ is the planetary mass, $R_{\mathrm{p}}$ is the planet's radius at the optical depth $\tau=2 / 3$ in the thermal wavelengths. ( $\tau$ is calculated using the grain-free Rosseland mean opacity $\kappa_{\text {th }}$ from Bell \& Lin (1994) and Freedman et al. $(2008)), F$ is the $\mathrm{X}$-ray flux in the wavelength range from 
1 to $20 \AA$ from Ribas et al. (2005), $\xi=R_{\text {roche }} / R_{\mathrm{p}}$, and

$$
K(\xi)=1-\frac{3}{2 \xi}+\frac{1}{2 \xi^{3}}
$$

accounts for the enhanced mass-loss rate by a factor of $1 / K(\xi)$ because the Roche lobe of a close-in planet can be close to the planet's surface (Erkaev et al. 2007). Lammer et al. (2009) discuss the possible values of the efficiency factor in energy-limited model and consider a realistic range of 0.1-0.25. Considering that it is the X-ray from 5 to $10 \AA$ responsible for heating (Owen \& Jackson 2012), the X-ray flux from 1 to $20 \AA$ used in our model might be too high. Thus, we set the nominal efficiency factor in the X-ray-driven regime to 0.1 . We also set it to 0.2 for a comparison population synthesis to generate larger mass-loss rates.

\subsection{EUV-driven Evaporation}

EUV photons $(h \nu \gtrsim 13.6 \mathrm{eV})$ can ionize the hydrogen in the upper atmosphere through the photoelectric effect. Because the $\mathrm{H}$ recombination cooling peak lies slightly above $10^{4} \mathrm{~K}$, photoionization-recombination will produce an equilibrium temperature of $\sim 10^{4} \mathrm{~K}$ in the ionized region (Dalgarno \& McCrav 1972). Based on the strength of the incoming EUV radiation, EUV-driven evaporation can be further divided into two sub-regimes (Murray-Clay et al. 2009):

At high EUV fluxes with $F_{\mathrm{EUV}}>F_{\text {crit }}$, a large fraction of the heating is lost to cooling radiation. The mass-loss rate increases slowly with the incoming flux as $\dot{M} \propto F_{E U V}^{0.6}$ (Murray-Clay et al. 2009). Therefore, the mass-loss rate cannot be estimated using a linear energy-limited equation of incident flux. Assuming that the escape flow is isothermal, the mass-loss rate is simply the mass flux at the sonic point in the flow (Murray-Clay et al. 2009), we have

$$
\dot{M}_{\mathrm{rr}-\lim } \sim 4 \pi \rho_{\mathrm{s}} c_{\mathrm{s}} r_{\mathrm{s}}^{2}
$$

where $c_{\mathrm{S}}=\left[k T /\left(m_{\mathrm{H}} / 2\right)\right]^{1 / 2}$ is the isothermal sound speed, $T=10^{4} \mathrm{~K}$ is the temperature at the sonic point, $r_{\mathrm{s}}=G M_{\mathrm{p}} /\left(2 c_{\mathrm{s}}^{2}\right)$ is the sonic point where the mass flow escapes the planet at the sound speed, and $\rho_{\mathrm{s}}$ is the flow density at the sonic point, which was calculated as described in Murray-Clay et al. (2009).

For $F_{\text {EUV }}<F_{\text {crit }}$, the mass-loss rate is calculated using an energy-limited model that is similar to Equation 1. The difference is that the $F$ used here is given by the EUV fluxes of a sun-like star from Ribas et al. (2005). $R_{\mathrm{p}}$ in the EUV-driven regime is set as the planetary radius where the optical depth becomes unity for EUV photons $(20 \mathrm{eV})$, at a pressure of $\sim 1$ nanobar (Murray-Clay et al. 2009). The efficiency factor is set by comparing Equation [1 with Equation 19 in Murray-Clay et al. (2009), which is an analytical equation used to estimate the mass-loss rates at a low $F_{\text {EUV }}$ and does not include the Roche lobe term $\xi \square$ The efficiency factor is 0.06 in the nominal evaporation model and 0.12 in the comparison population synthesis.

1 The Roche lobe term is also neglected in the energy-limited EUV- driven regime in our evaporation model.
We adopt the criterion from Murray-Clay et al. (2009), which states that, for $F_{\mathrm{EUV}}>10^{4} \mathrm{erg} \mathrm{cm}^{-2} \mathrm{~s}^{-1}$, evaporation is in the radiation-recombination-limited regime for hot Jupiters orbiting main-sequence solar analogs. In reality, the critical $F_{\mathrm{EUV}}\left(F_{\text {crit }}\right)$ for the transition from a radiation-recombination- to an energy-limited evaporation is a function of the planetary mass and radius, among other characteristics. In our work, we simply choose a constant $F_{\text {crit }}=10^{4} \mathrm{erg} \mathrm{cm}^{-2} \mathrm{~s}^{-1}$ for each planet. The variations of this critical value and its influence on the final planet population will be described in $\S 3.5$.

\subsection{Transition Between the X-ray- and EUV-driven Regimes}

Whether evaporation is X-ray- or EUV-driven depends on the intensity of the EUV and X-ray irradiation received by a planet. We use the criterion from Owen \& Jackson (2012), which separates X-ray- and EUV-driven regimes based on whether the X-ray-driven flow can reach the sonic point before it enters the ionization front. This criterion can be determined based on the EUV luminosity of the host star. The threshold EUV luminosity for the escape flow in an EUV-driven regime is (Owen \& Jackson 2012):

$$
\begin{aligned}
\Phi_{*} \geq & 10^{40} \mathrm{~s}^{-1}\left(\frac{a}{0.1 \mathrm{AU}}\right)^{2}\left(\frac{\dot{m}_{\mathrm{X}}}{10^{12} \mathrm{~g} \mathrm{~s}^{-1}}\right)^{2}\left(\frac{A}{1 / 3}\right) \\
& \times\left(\frac{\beta}{1.5}\right)\left(\frac{R_{\mathrm{p}}}{10 R_{\oplus}}\right)^{-3} .
\end{aligned}
$$

where $\Phi_{*}$ is the EUV luminosity (in photons per second) of the host star, $A$ (typically $\approx 1 / 3$ ) is a geometric factor that approximates the steepness of the density fall-off in the ionized portion of the X-ray-heated flow (Johnstone et al. 1998), $\beta$ is the ratio of the X-ray sonic surface to the planetary radius (it is of order unity (Owen \& Jackson 2012); we set it to 1.5 herein), and $\dot{m}_{\mathrm{X}}$ is the mass flux of the X-ray-driven flow that enters the ionization front.

\section{PLANETARY EVOLUTION}

\subsection{The Synthetic Planet Populations}

The synthetic planet populations adopted herein are based on a planet formation model (Alibert et al. 2005; Mordasini et al. 2012a, b) , in which we simulate the accretion of solid/gas materials and the disc-based migration of a planet in combination with the evolution of a protoplanetary gas disk. At $8 \mathrm{Myr}$, nearly all protoplanetary gas disks have disappeared (Mordasini et al. 2009); thereafter, the planets enter the evolution stage, where both gas accretion and disk-driven migration end. Planet-planet scattering and Kozai migration is not included in the model because we use the one-embryo-perdisk approximation. We take a snapshot of each planet in a population at $10 \mathrm{Myr}$ and set it as the initial condition of the evolution stage. We use the following parameters for each planet: core mass, envelope mass, luminosity, mass-fraction of the ice in the core, initial deuterium fraction, and semi-major axis.

The population-wide effect of atmospheric evaporation is our main focus. We consider two variable quantities 
in population synthesis: one is the description of the evaporation model that varies with the dominant heating source (X-ray, EUV, or both) and heating efficiency, and the other is the ISM grain opacity reduction factor used during the formation stage. The grain opacity during the formation phase controls the amount of $\mathrm{H} / \mathrm{He}$ a core of given mass and luminosity can accrete (Movshovitz et al. 2010; Mordasini et al. 2014a). We follow the evolution of different synthetic planet populations using different evaporation models. The details for each simulation are listed in Table 1 All populations are formed around a 1 $M_{\odot}$ star and using the one-embryo-per-disk approximation. A description of the concurrent formation of several planets can be found in Alibert et al. (2013). We define the nominal evaporation model as one that includes both X-ray-and EUV-driven regimes. The nominal synthetic population is defined as a population calculated using the isothermal type I migration rate (Tanaka et al. 2002 ) with a reduction factor of 0.1 and a grain opacity reduction factor $\left(f_{\text {opa }}\right)$ of 0.003 compared with the full ISM value. The low-mass, close-in planets in this population are formed based on a protoplanetary disk that includes the stellar irradiation for calculating the disk's temperature structure, and the combined viscous and thermal criteria for the transition from type I to type II migration. These assumptions are more realistic than the assumptions in Mordasini et al. (2009), wherein nonirradiated disks were used with only the thermal criterion and a type I migration rate with a reduction factor of 0.001 . The inner boundary of the disk in our model is 0.1 AU due to limitations in the stellar irradiation description for the disk (Fouchet et al. 2012). Radial velocity and transit surveys have detected many planets within $0.1 \mathrm{AU}$, which demonstrates that even close-in planets with orbital periods less than 10 days are common (Mayor et al. 2011; Dong \& Zhu 2013; Petigura et al. 2013a). Planets can reach close-in orbits through diskdriven migration (Goldreich \& Tremaine 1980; Lin et al. 1996; Zhou et al. 2005) or migration due to planetesimal disk dynamics (Terquem \& Papaloizou 2007; Ji et al. 2011; Ormel et al. 2012). A close-in planet may also be formed through Kozai migration with tidal circularization (Wu \& Murray 2003; Fabrycky \& Tremaine 2007), but this process can take considerably longer, and the intense atmospheric evaporation stage may have passed. Because the inner boundary $(0.1 \mathrm{AU})$ in the semi-major axis distribution of our planet populations is too large to reasonably study hydrodynamic evaporation, we manually shift the semi-major axes of all planets inward by $0.04 \mathrm{AU}$; thus, the planet populations begin at $0.06 \mathrm{AU}$.

\subsection{Planet Structure Model}

A planets structure consists of a core and a gaseous envelope. The planetary core mass is constant during evolution. The core radius is determined by its mass, its mass-fraction of ice, and the pressure at the bottom of the gaseous envelope (Mordasini et al. $2012 \mathrm{~b})$. We assume spherical symmetry in the planetary envelope and solve its structure by combining the following one-dimensional hydrostatic equations:
TABLE 1

Details of THE DifFERENT Simulations

\begin{tabular}{lccc}
\hline $\begin{array}{l}\text { Simulation } \\
\text { run }\end{array}$ & $\begin{array}{c}\text { Type I migration } \\
\text { rate }\end{array}$ & $f_{\text {opa }}$ & Evaporation model \\
\hline XE & $0.1 \times$ isothermal-rate & 0.003 & X-ray + EUV \\
NoEV & $0.1 \times$ isothermal-rate & 0.003 & No Evap \\
SatE & $0.1 \times$ isothermal-rate & 0.003 & EUV (Saturation) \\
XE2 & $0.1 \times$ isothermal-rate & 0.003 & XE $\times 2$ \\
L12 & $0.1 \times$ isothermal-rate & 0.003 & Lopez et al. $(2012)$ \\
B04 & $0.1 \times$ isothermal-rate & 0.003 & Baraffe et al. $(2004)$ \\
\hline NIOpa003 & non-isothermal & 0.003 & X-ray + EUV \\
NIOpa0 & non-isothermal & 0.0 & X-ray + EUV \\
NIOpa1 & non-isothermal & 1.0 & X-ray + EUV \\
\hline
\end{tabular}

$$
\begin{array}{ccc}
\frac{\mathrm{d} m}{\mathrm{~d} r}=4 \pi r^{2} \rho & (5 \mathrm{a}) & \frac{\mathrm{d} \tau}{\mathrm{d} r}=\kappa_{\mathrm{th}} \rho \\
\frac{\mathrm{d} P}{\mathrm{~d} r}=-\frac{G m}{r^{2}} \rho & (5 \mathrm{~b}) & \frac{\mathrm{d} L}{\mathrm{~d} r}=0
\end{array}
$$

where $r$ is the radius as measured from the planetary center, $m$ is the cumulative mass inside $r, \rho$ is the density in each spherical shell, $P$ the pressure, $G$ the gravitational constant, and $L$ is the planetary luminosity, which includes radiogenic heating from the solid core. We assume that the luminosity is constant with radius, which does not significantly affect the evolution as discussed in Mordasini et al. (2012a).

The temperature gradient in the gaseous envelope depends on both the optical depth and heat transfer mechanism (convective or radiative) at each envelope layer. We separate the gaseous envelope into two parts: the atmosphere where most of the stellar irradiation is absorbed and the envelope that lies below the atmosphere. If an atmospheric layer is convectively stable, we adopt the globally averaged temperature profile from Equation (49) in the semi-grey model of Guillot (2010) (which is derived using the Eddington approximation):

$$
\begin{aligned}
T^{4}= & \frac{3 T_{\mathrm{int}}^{4}}{4}\left\{\frac{2}{3}+\tau\right\}+\frac{3 T_{\mathrm{eq}}^{4}}{4}\left\{\frac{2}{3}+\right. \\
& \left.\frac{2}{3 \gamma}\left[1+\left(\frac{\gamma \tau}{2}-1\right) e^{-\gamma \tau}\right]+\frac{2 \gamma}{3}\left(1-\frac{\tau^{2}}{2}\right) E_{2}(\gamma \tau)\right\}
\end{aligned}
$$

where $T_{\text {int }}=\left(L /\left(4 \pi \sigma_{B} R_{\mathrm{p}}^{2}\right)\right)^{1 / 4}$ is the intrinsic temperature that characterizes the heat flux from the planet's interior $\left(\sigma_{B}\right.$ is the Stefan-Boltzmann constant), $T_{\text {eq }}=$ $T_{*}\left(R_{*} /(2 D)\right)^{1 / 2}$ is the equilibrium temperature obtained by averaging the stellar radiation over the entire planet surface ( $T_{*}$ is the stellar temperature, $R_{*}$ is the stellar radius, and $D$ is the distance from the planet to the star), $\gamma=\kappa_{\mathrm{v}} / \kappa_{\mathrm{th}}$ is the ratio of the visible opacity to the thermal opacity (Guillot 2010). The visible opacity $\kappa_{\mathrm{v}}$ is not explicitly calculated but is incorporated in the model by $\gamma . E_{2}(\gamma \tau)$ is the exponential integral $E_{n}(z) \equiv \int_{1}^{\infty} t^{-n} e^{-z t} d t$ with $n=2$.

The boundary between the atmosphere and envelope should be at the optical depth in visible wavelengths $\tau_{\mathrm{v}} \gg 1$. Based on the $\gamma$ defined in the semi-grey model, we have $\tau \gg 1 /(\sqrt{3} \gamma)$ at the transition (Rogers et al. 2011). Because most of the starlight is absorbed at pressures less than 10 bar (Guillot \& Showman 2002), we set 
TABLE 2

THE $\gamma=\kappa_{\mathrm{v}} / \kappa_{\text {th }}$ USED IN THE ATMOSPHERIC MODEL.

\begin{tabular}{cc}
\hline Temperature $(\mathrm{K})$ & $\gamma$ \\
\hline 260 & 0.005 \\
388 & 0.008 \\
584 & 0.027 \\
861 & 0.07 \\
1267 & 0.18 \\
1460 & 0.19 \\
1577 & 0.18 \\
1730 & 0.185 \\
1870 & 0.2 \\
2015 & 0.22 \\
2255 & 0.31 \\
2777 & 0.55 \\
\hline
\end{tabular}

the atmosphere/envelope boundary at $\tau=100 /(\sqrt{3} \gamma)$ (which corresponds to a pressure of $\sim 10$ bar). If the envelope at $\tau>100 /(\sqrt{3} \gamma)$ is convectively stable, the radiative temperature gradient is calculated using the diffusion approximation that only includes the planet's intrinsic luminosity:

$$
\frac{\mathrm{d} T}{\mathrm{~d} r}=-\frac{3 \kappa_{\mathrm{th}} \rho L}{64 \pi \sigma_{B} T^{3} R^{2}}
$$

On the other hand, if a layer is convectively unstable (i.e., the adiabatic temperature gradient is less steep than the radiative temperature gradient), we use the adiabatic temperature profile instead:

$$
\frac{\mathrm{d} T}{\mathrm{~d} r}=\frac{T}{P} \frac{\mathrm{d} P}{\mathrm{~d} r}\left(\frac{\ln T}{\ln P}\right)_{\mathrm{ad}}
$$

where the adiabatic temperature gradient is calculated using the equation of state of Saumon et al. (1995). This convective adjustment is also used in the atmosphere, although we do not allow detached convective zones there.

Planetary evolution is modeled using the framework of Mordasini et al. (2012a, b) , wherein the planetary luminosity $L$ and its temporal evolution is derived through energy conservation as $L=-\mathrm{d} E_{\text {tot }} / \mathrm{d} t$, where $\mathrm{d} E_{\text {tot }}$ is the energy change due to gravitational contraction and release of internal heat (neglecting planetary rotation). The luminosity $L$ at each timestep controls the planetary structure and the changes of the interior adiabat, and hence the temporal evolution of the planet.

\subsection{Atmosphere Calibration}

One important parameter in the semi-grey model, $\gamma$, determines how much of the incoming flux is absorbed in the upper atmosphere. The $\gamma$ values used in our simulation are shown in Table 2 and are determined by comparing the temperature in the deep isothermal zone of the analytical model (Equation 6) with the results from the line-by-line radiative transfer models (Fortney et al. 2005, 2008; Parmentier et al. 2013b). The line-by-line EGP (Extrasolar Giant Planet) code was initially developed by McKay et al. (1989) to study Titan's atmosphere. Since then, it has been extensively modified and adapted for studies on giant planets (Marley \& McKav 1999), brown dwarfs (Marley et al. 1996, 2002; Burrows et al. 1997), and hot Jupiters (e.g., Fortney et al. 2005, 2008; Showman et al. 2009).

The $\gamma$ at a specific equilibrium temperature can be

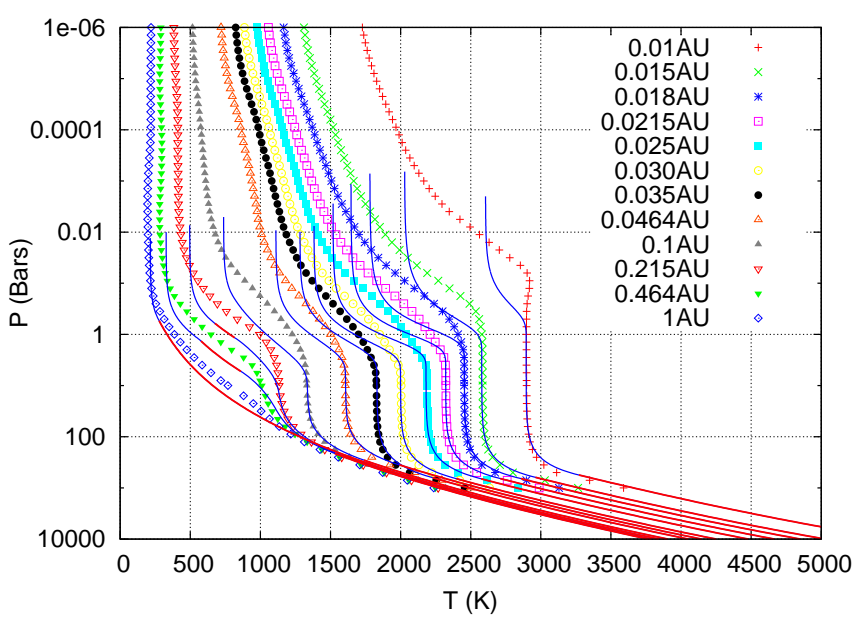

FIG. 1.- Pressure-temperature models from Fortney et al. (2008) (points) and the corresponding analytical fits (solid line) for different semi-major axes. The modeled planets are with $g=15 \mathrm{~m}$ $\mathrm{s}^{-2}$ and $T_{\mathrm{int}}=200 \mathrm{~K}$, and they orbit around a sun-like star. The discrepancy in the upper part of atmosphere is due to the non-grey effects (Parmentier \& Guillot 2014; ; Parmentier et al. [2013b).

determined through interpolation between these tabulated values. Figure 1 shows the PT profiles obtained by two different models. The numerical profiles are calculated assuming a clear-sky, solar composition atmosphere where $\mathrm{TiO}$ and $\mathrm{VO}$ have rained out of the atmosphere (see Parmentier et al. 2013a; Parmentier \& Guillot 2014) and is neglected in the model. However, the effects of a non-solar composition may be important but are not considered in this study. The analytical solution is highly consistent with the line-by-line model in the deep atmosphere. For pressures lower than 1 bar, the discrepancy is due to non-grey effects (Parmentier \& Guillot 2014).

\subsection{Mass-radius Relationship of Low-Mass Planets}

We then compare the mass-radius relationships of the low-mass planets from our model with those of Rogers et al. (2011). These low-mass planets have different envelope mass fractions $f_{\text {env/tot }}$ (the ratio of the envelope mass to the total planetary mass) and are at an equilibrium temperature of 500 or $1000 \mathrm{~K}$. Rogers et al. (2011) use a fixed $\gamma$ of $0.6 \sqrt{T_{\text {irr }} / 2000 \mathrm{~K}}$ based on the fit in Guillot $(2010)$, where $T_{\mathrm{irr}}=T_{*}\left(R_{*} / D\right)^{1 / 2}$ is the irradiation temperature. Parmentier \& Guillot (2014) examine the fitted $\gamma=0.6 \sqrt{T_{\mathrm{irr}} / 2000 \mathrm{~K}}$ and find that it is poor for temperatures lower than $1000 \mathrm{~K}$ due to the disappearance of absorption by alkaline molecules (sodium, potassium, etc.). Rogers et al. (2011) also set the planetary boundary at an optical depth of $\tau=2 / 3$. As shown in Figure 2. the planetary radius is closely related to the $\gamma$ value and the planetary boundary. The mass-radius relationships calculated using the same $\gamma$ and planetary boundary as Rogers et al. (2011) are highly consistent with their results. We also show two other groups of mass-radius relationships that are calculated using the tabulated $\gamma$ at 500 and $1000 \mathrm{~K}$. In addition to the different $\gamma$, the planetary boundary for these two groups is set at $\tau=0.01$ (i.e., the atmospheric structure is integrated from $\tau=0.01$; notably, we adopt the radius at the optical depth $\tau=2 / 3$ as the planetary radius, and for the figures herein, the plotted planetary radii are at $\tau=2 / 3$ ). These 

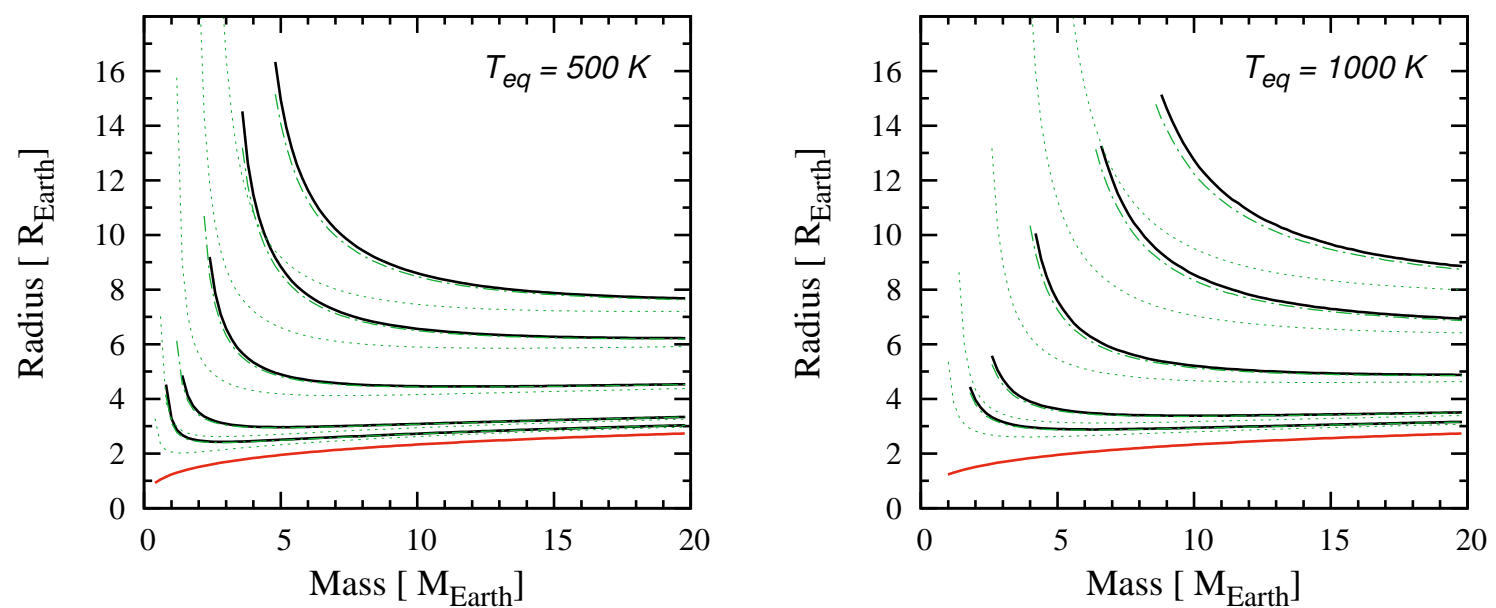

FIG. 2.- Mass-radius relationships for low-mass planets with different envelope fractions $f_{\text {env } / \text { tot }}\left(M_{\text {env }} / M_{\mathrm{p}}\right)$. The radius plotted here are the planetary radii at $\tau=2 / 3$. There are three groups of lines: The green dotted lines are calculated using the fixed $\gamma=0.6 \sqrt{T_{\mathrm{irr}} / 2000 \mathrm{~K}}$ and the planetary boundary at $\tau=2 / 3$; these setting are the same as Rogers et al. (2011). The other two groups use the interpolated $\gamma$ at equilibrium temperatures at 500 and $1000 \mathrm{~K}$, and the planetary boundary at $\tau=0.01$ (the planetary radii plotted in the figure are at $\tau=2 / 3$ ). The green dot-dashed lines use the isotropic temperature profile (Equation 27 in Guillot (2010)), it is for comparison with the thick black lines that use the globally averaged temperature profile (Equation 49 in Guillot (2010)). From bottom to top, the $f_{\text {env }}$ tot in each group is $0.001,0.01,0.1,0.3$, and 0.5 , respectively. The red line at the bottom is the mass-radius relationship of the solid cores for planets with $f_{\text {env } / \text { tot }}=0.001$. In all of the runs, the luminosity of each planet is set according to $L_{\mathrm{p}} / M_{\mathrm{p}}=10^{-10.5} \mathrm{~W} \mathrm{~kg}{ }^{-1}$.

two groups of mass-radius relationships are considerably different from those of Rogers et al. (2011), especially the low-mass planets with a high $f_{\text {env/tot }}$. The difference between these two groups is the temperature profile used in the semi-grey model in the upper atmosphere. One group uses the isotropically averaged temperature profile in Rogers et al. (2011), and the other group uses the globally averaged temperature profile, which considers the advective transport of energy to a certain extent (Guillot 2010). In contrast to the large discrepancies from the different $\gamma$ and planetary boundary, the differences between the mass-radius relationships created by the isotropically averaged temperature profile and the globally averaged profile are small. The planetary radii calculated using the globally averaged temperature profile are slightly larger than the radii calculated using the isotropic profile. For the equilibrium temperature $500 \mathrm{~K}$ and with an $f_{\text {env/tot }}$ of 0.1 , the radius of a $3 M_{\oplus}$ planet calculated using the globally averaged temperature profile is $\sim 4 \%$ larger than the radius for the isotropic profile, and the difference between the radii from the two temperature profiles is $<1 \%$ for planets larger than 6 $M_{\oplus}$. The isotropic temperature profile is only used for the comparison group shown in Figure 2. In our population syntheses, planetary evolution is calculated using the globally averaged temperature profile.

The red lines in Figure 2 show the radii of the solid cores of the planets with an $f_{\text {env/tot }}$ of 0.001 . The large differences between the radii of the cores and the total planetary radii show that the planetary envelope with only $0.1 \%$ of the planetary mass produces a large increase in the planetary radius, which is a well-known effect (e.g., Adams et al. 2008). For example, at an equilibrium temperature of $500 \mathrm{~K}$, a planet with a total mass of approximately $1 M_{\oplus}$ and an envelope of $0.1 \%$ of the planetary mass will have a radius that is greater than 2 $R_{\oplus}$. The planet's atmosphere is bloated due to its low surface gravity and the heating from the incoming irradiation. Consequently, the planetary radius will decrease dramatically after its entire envelope is removed through evaporation.

\subsection{Illustrative Model Runs: Planetary Evolution with Escape}

Figure 3 shows the evolution of a Neptunian planet and a Jovian planet located at 0.03 AU. The atmospheric escape is included such that the planetary envelope mass is reduced at each timestep based on the calculated massloss rate.

The Neptunian planet, with a $15 M_{\oplus}$ core and $10 M_{\oplus}$ envelope, loses its entire initial envelope in the first 220 Myr when both X-ray- and EUV-driven evaporation are included. If only EUV-driven evaporation is included, the planet can retain an envelope of $\sim 2.9 M_{\oplus}$ after 10 Gyr of evolution. X-ray-driven evaporation in the early stage of planetary evolution is efficient at removing gas from a planet because the planetary atmosphere is bloated and the X-ray flux from the host star is intense (Ribas et al. 2005). The mass-loss rates in the first $1 \mathrm{Myr}$ of planetary evolution are approximately $4 \times 10^{-6} M_{\oplus} / y r$. From $\sim 1.45 \times 10^{8}$ years and forward, evaporation transitions to the EUV-dominated radiation-recombinationlimited regime. At this time, the planet only has a 0.085 $M_{\oplus}$ envelope remaining. Soon thereafter, the planet 

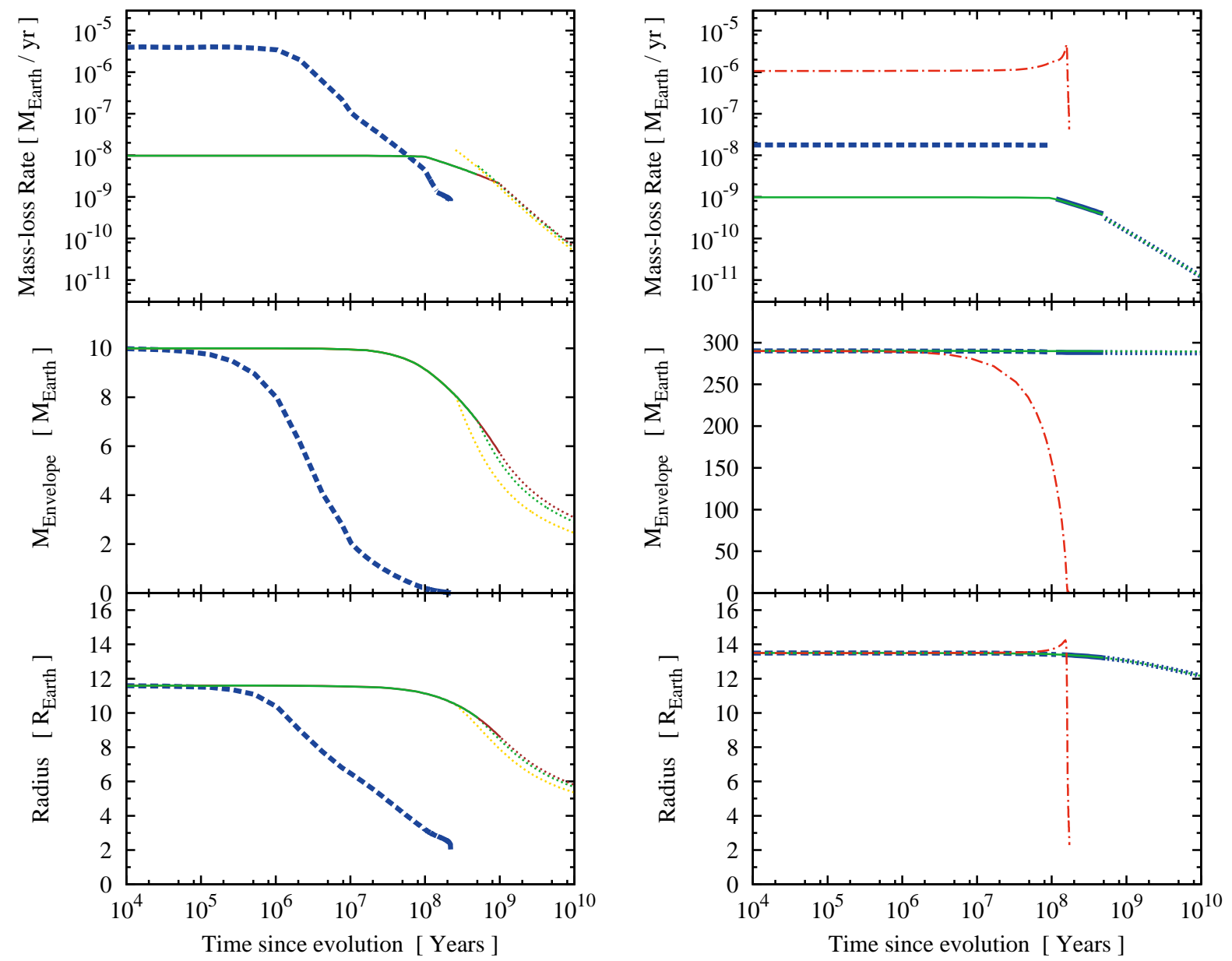

FIG. 3. - Temporal evolution of two close-in planets at 0.03 AU. The left column shows the evolution of a Neptunian planet with a 15 $M_{\oplus}$ core and a $10 M_{\oplus}$ envelope. The right column shows the evolution of a Jovian planet with a $25 M_{\oplus}$ core and a $290 M_{\oplus}$ envelope. The thick blue line is the experiment includes both X-ray- and EUV-driven evaporation; the dashed part of the line indicates the X-ray-driven regime, the solid part indicates the radiation-recombination-limited EUV-driven regime, and the dotted part indicates the energy-limited EUV-driven regime. The thin green, yellow, and brown lines are the experiments that include only EUV-driven evaporation; the solid part indicates the radiation-recombination-limited regime, and the dotted part indicates the energy-limited regime. The yellow and brown lines show the evolution of the Neptunian planet using the $F_{\text {cirt }}$ from a radiation-recombination-limited to an energy-limited regime of $2 \times 10^{4} \mathrm{erg} \mathrm{cm}^{-2} \mathrm{~s}^{-1}$ and $0.5 \times 10^{4} \mathrm{erg} \mathrm{cm}^{-2} \mathrm{~s}^{-1}$. The red dash-dotted lines in the right column use the energy-limited model with a $100 \%$ heating efficiency (Baraffe et al. 2004),

loses its entire envelope and becomes a $15 M_{\oplus}$ rocky core with $\mathrm{a} \sim 2 R_{\oplus}$ radius. In contrast, if only EUV-driven evaporation is included, in its early stage, the planet is in the radiation-recombination-limited regime wherein most incoming energy is lost to cooling radiation. The mass-loss rates in this regime are determined based on the density of the escape flow at the sonic point; they are below $10^{-8} M_{\oplus} / y r$ in the first 100 Myr of planetary evolution. The transition from radiation-recombinationlimited evaporation to energy-limited evaporation occurs at approximately $5.1 \times 10^{8}$ years and when the EUV flux from the star is below $10^{4}$ erg $\mathrm{cm}^{-2} \mathrm{~s}^{-1}$. After $10 \mathrm{Gyr}$, the final bulk composition of this planet includes a $15 M_{\oplus}$ core with a $2.9 M_{\oplus}$ envelope, and the planetary radius is $5.67 R_{\oplus}$. The discontinuous change in the mass-loss rate for this experiment demonstrates that the critical EUV flux, which delimits the radiation-recombination-limited and energy-limited evaporation regimes, is in principle inappropriate for this Neptunian planet. In Figure 3. we also show the evolution of the same planet but using different $F_{\text {crit }}$ at $2 \times 10^{4}$ and $0.5 \times 10^{4}$ erg cm $\mathrm{cm}^{-2}$. These two experiments generate a final planetary radius of $5.34 R_{\oplus}\left(F_{\text {crit }}=2 \times 10^{4} \mathrm{erg}_{\mathrm{cm}^{-2}} \mathrm{~s}^{-1}\right)$ and $5.81 R_{\oplus}$ $\left(F_{\text {crit }}=0.5 \times 10^{4} \mathrm{erg} \mathrm{cm}^{-2} \mathrm{~s}^{-1}\right)$, which corresponds to the $5.8 \%$ and $2.5 \%$ changes in the radius, respectively. Notably, these two experiments only include EUV-driven evaporation. When X-ray-driven evaporation is included in the model, most of the planets that could be evaporated to bare cores lost their entire envelope when the evaporation remained in the X-ray-driven regime. Thus, the constant $F_{\text {crit }}$ used for the low-mass planets in our model will not excessively affect the population-wide radius distribution, as shown in the following section.

For the Jovian planet, the evaporation models with or without X-ray-driven mass-loss do not show significantly different final results. The modeled planet has a $25 M_{\oplus}$ 

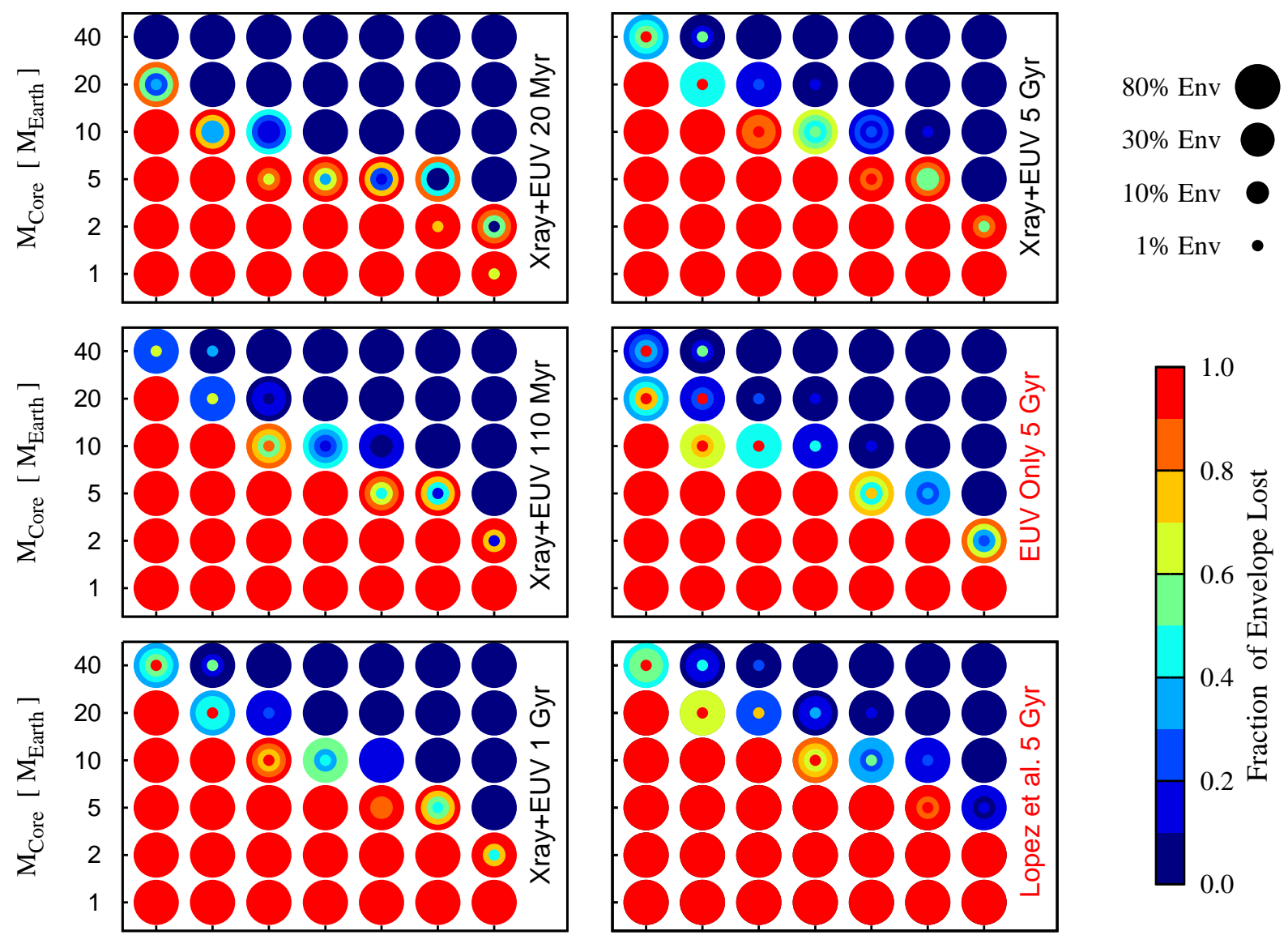

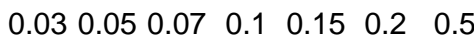

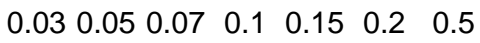

Semi-major Axis [AU]

Semi-major Axis [AU]

FIG. 4.- A parameter study of planetary evolution coupled with evaporation in the $M_{\text {core }}$ versus semi-major axis plane. All planets orbit around a $1 M_{\odot}$ star. There are four planets (different point sizes) with different choices of $f_{\text {env } / \text { core }}$ at each grid node, as indicated in the top right corner of the figure. The color of each point shows how much of the initial envelope was lost. The left column and the top panel in the right column show the temporal evolution of the simulation using the nominal evaporation model that includes both X-rayand EUV-driven regimes. The three panels in the right column compare the final results of three simulations using different evaporation models: both X-ray- and EUV-driven are included (nominal), EUV-driven only, and the energy-limited model from Lopez et al. (2012).

core and a $290 M_{\oplus}$ envelope. As shown in the right column of Figure 3. the evolution of this planet under different evaporation models overlaps during the 10 Gyr's evolution. The only notable difference between the two runs is that, in the first $100 \mathrm{Myr}$, the mass-loss rate in the X-ray-driven regime is approximately 18-fold greater than in the EUV-driven, radiation-recombination-limited regime. This difference is insufficient for producing a notable change in the planetary mass and radius because the total mass of the envelope lost only composes a small fraction of the planetary mass. In the end, the experiment that also included X-ray-driven evaporation lost $\sim$ $2.29 M_{\oplus}$ of the envelope, and the other run, which did not include X-ray-driven evaporation, lost $\sim 0.72 M_{\oplus}$ of the envelope. The only way for a Jovian planet to lose a significant portion of its envelope is by assuming an energy-limited evaporation model with a $100 \%$ heating efficiency (Baraffe et al. 2004). In this case, the Jovian planet began with a mass-loss rate greater than $10^{-6} M_{\oplus} \mathrm{yr}^{-1}$. With this high mass-loss rate, the planetary radius expands at approximately $10^{8}$ years due to the entropy change in the outer radiative layers. Detailed descriptions of this interesting process can be found in Baraffe et al. (2004); Hiellming \& Webbink (1987). In turn, the larger planetary radius results in an even greater mass-loss rate. Beginning at $\sim 1.5 \times 10^{8}$ years, the planet reaches a runaway mass-loss stage, as observed by Baraffe et al. (2004). Eventually, the planet loses its entire initial envelope after $\sim 1.72 \times 10^{8}$ years of evolution.

\subsection{A Parameter Study}

In Figure 4, we show a parameter study on evaporation. We consider three parameters: the planetary semimajor axis, the planetary core mass, and the ratio of the planetary envelope mass to the core mass $\left(f_{\text {env } / \text { core }}\right)$. The parameter space is similar to Lopez \& Fortney (2013), where the incident flux from the star is used instead of the planetary semi-major axis because the distance from 
a planet to its host star essentially determines the incident flux that the planet can receive for a fixed stellar type. The modeled planets are located at 0.03-0.5 AU with a core of 1-40 $M_{\oplus}$. At each semi-major axis and each core mass, four planets have different $f_{\text {env/core }}$, i.e., $1 \%, 10 \%, 20 \%$, and $80 \%$. We do not consider planets with a larger $f_{\text {env/core }}$. Due to the long Kelvin-Helmholtz timescale, it is unlikely that a small core will accrete significant levels of gas during the formation stage. A massive core may accrete high levels of gas, but the effect of evaporation on gas giants is small, as shown in Figure 3. thus, gas giants are not included in this parameter study. Because all planets in this parameter study are artificial bodies without an initial luminosity from the self-consistent planet formation stage, we set their initial luminosities to the value that corresponds to an entropy at the core-envelope boundary that equals $7.11 \times\left(M_{\text {core }} / M_{\oplus}\right)^{0.0422} \times\left(f_{\text {env/core }}\right)^{0.0175}$. This is an empirical fit of the central entropies of the planets with a $1-40 M_{\oplus}$ core and a $f_{\text {env/core }}$ of $1 \%-80 \%$ in our synthetic population at $10 \mathrm{Myr}$. This fit shows that the initial entropy of a planet increases with the core mass and envelope to core mass fraction. The initial entropy of low-mass planets is an interesting subject that will be investigated separately in future work.

The left column of Figure 4 shows the evolution of the simulation using the nominal evaporation model, which includes both the X-ray- and EUV-driven mechanisms. Four snapshots of this simulation (at $20 \mathrm{Myr}, 110 \mathrm{Myr}, 1$ Gyr, and $5 \mathrm{Gyr}$ ) are presented. At $20 \mathrm{Myr}$ (after $10 \mathrm{Myr}$ of evolution), all of the planets in the bottom left corner have lost their entire envelopes and have become bare rocky cores. There are two reasons for this observation: one, the large planetary radii due to the heating effect from the intense stellar irradiation and large planetary intrinsic entropies during the early stage and, two, the manually fixed $f_{\text {env/core }}$ for small cores can be substantially higher than predicted in the formation calculations. In particular, the formation calculations do not produce cores of 1,2 , or $5 M_{\oplus}$ with a $f_{\text {env/core }}$ of $80 \%$ at 0.03 or $0.05 \mathrm{AU}$. The high temperature at the outer boundary and large initial luminosities for these small planets with a large $f_{\text {env/core }}$ appear to produce an unstable envelope structure that cannot be modeled using the hydrostatic equilibrium approximation. The only structure that we find for these planets is a bloated atmosphere that expands beyond the Hill sphere, which is unstable. Thus, their initial atmospheres evaporate in a short time. Kurokawa \& Nakamoto (2014) show comparable cases with low-mass planets undergoing a similar dynamic, i.e., runaway mass-loss. After the early, intense, $\mathrm{X}$-ray-driven evaporation stage, the evaporation transitions to the moderate radiation-recombination-limited or energy-limited EUV-driven regimes and the snapshots at the 1 Gyr and 5 Gyr only slightly differ.

The three panels in the right column compare the final status of the three simulations, with the only difference between them being the evaporation model. One uses the nominal model, which includes both X-ray- and EUVdriven mechanisms, another only uses the EUV-driven evaporation model, and a third uses the same energylimited evaporation model as that in Lopez et al. (2012). The final configurations of these three groups are simi- lar: the planets in the bottom left corner become bare rocky cores, while planets with large cores at large semimajor axes retain most of their initial envelopes. The differences between these three groups lie in the diagonal region of each panel. X-ray-driven evaporation in the early stage is more effective at removing planetary atmosphere than EUV-driven evaporation; thus, the diagonal region in the simulation using the nominal evaporation model includes more bare cores than the simulation using only the EUV-driven mechanism. The simulation using the energy-limited evaporation model in Lopez et al. (2012) produces the greatest mass-loss. Consequently, it includes the fewest planets with portions of their initial envelopes, and the $f_{\text {env/core }}$ of each planet at $5 \mathrm{Gyr}$ is the smallest of the three simulations.

\section{THE POPULATION-WIDE IMPACT OF EVAPORATION}

We then couple planetary population synthesis with different evaporation models to determine the population-wide effect of atmospheric evaporation. First, in Figure 5, 6, and 7. we show the evolution of the mass-loss rates, mass and radius distribution of our nominal planetary population using the nominal evaporation model.We then investigate the influence of the efficiency of evaporation mechanism. In Figure 8, 9, 10, and 11. we compare the mass-loss rates, mass and radius distributions, and mass-radius relationships using different evaporation models (or without evaporation). The effect of the different grain opacities used during the planetary formation stage is demonstrated by the mass-radius relationships of three different planet populations in Figure 12. Finally, we compare the radius distribution of our synthetic populations with the Kepler data in Figure 13 and 14.

\subsection{Synthetic Planets: A, the Reference Simulation}

In Simulation XE (see Table 1), we apply the nominal evaporation model (X-ray + EUV) to the nominal planetary population; therefore, it is referred to as our reference simulation. In Figure [5] we plot the temporal evolution of the planet mass-loss rates for Simulation XE in the planet's semi-major axis versus mean density plane. The evaporation rates of close-in planets are large at 0.02 Gyr and 0.11 Gyr. At 0.02 Gyr, close-in planets at 0.06 AU can have a mass-loss rate of $\sim 10^{-6} M_{\oplus} \mathrm{yr}^{-1}$. The mass-loss rates for most of planets beyond $1 \mathrm{AU}$ are less than $10^{-10} M_{\oplus} \mathrm{yr}^{-1}$. At $1 \mathrm{Gyr}$, nearly all planets that retain portions of their envelope have a mass-loss rate of less than $10^{-10} M_{\oplus} \mathrm{yr}^{-1}$, which explains why the envelope mass fractions and radius distributions in Figure 6 and Figure 7 (in the following section) barely change after 1 Gyr. The parallel dashed lines show identical massloss rates for a purely energy-limited evaporation model (i.e., the planets along each line have the same mass-loss rate as those in the energy-limited regime); the mass-loss rate is a function of the planets mean density and incoming flux. We use an energy-limited model in both the X-ray and the low EUV regimes; thus, most of the color belts in Figure 5] are parallel to these dashed lines. At $0.02 \mathrm{Gyr}$, a considerable portion of the planets are in the radiation-recombination-limited regime at $\sim 0.2$ to $1 \mathrm{AU}$; hence, the yellow and orange belts slightly deviate from the parallel dashed lines. From 1 Gyr, nearly all of the 

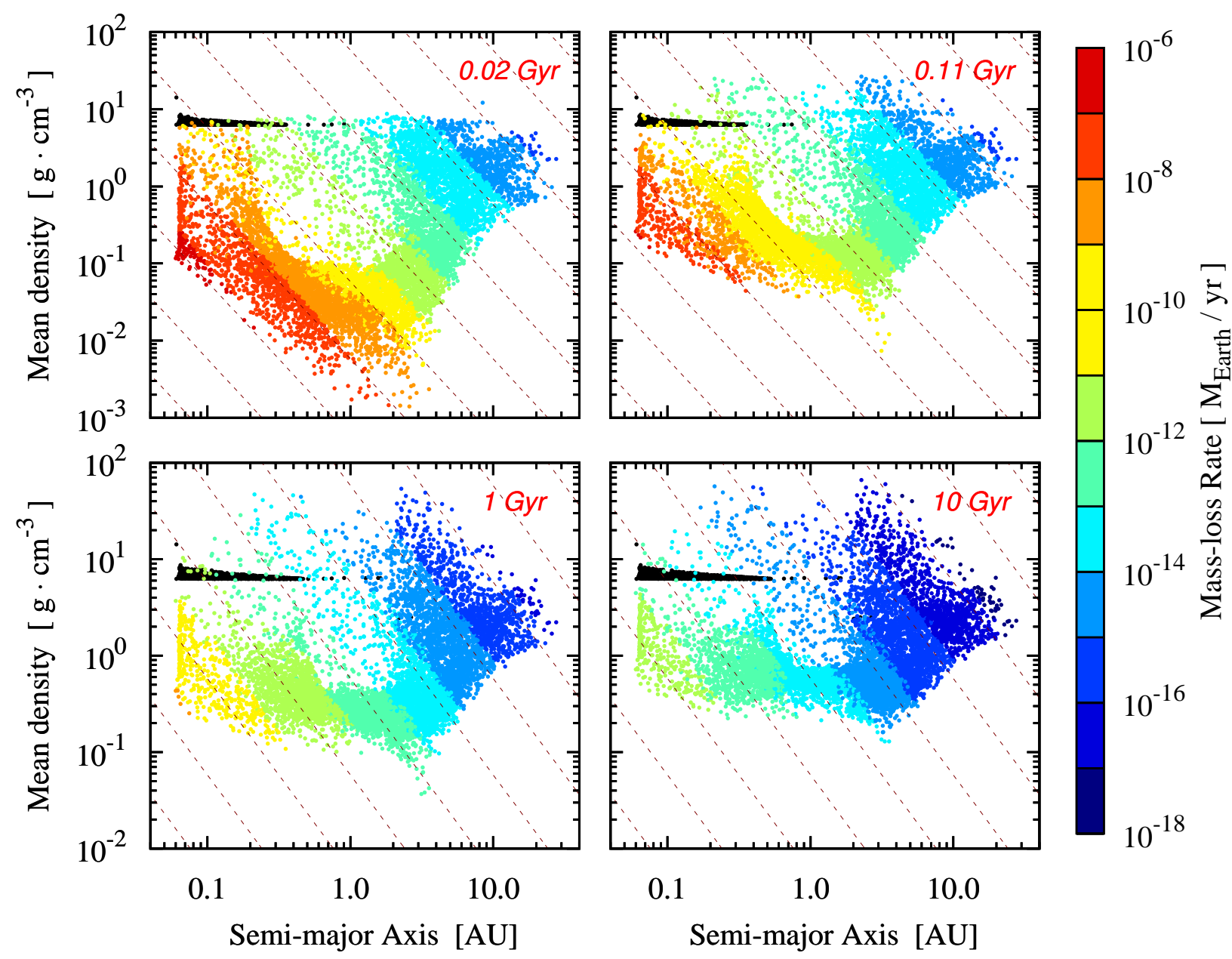

FIG. 5.- Temporal evolution of the mass-loss rates of the reference simulation in the planetary semi-major axis versus mean density plane. All planets orbit around a $1 M_{\odot}$ star. Each point in the figure corresponds to a planet in the synthetic population. The color of each point show the mass-loss rate of the planet. The black points are the planets that have lost all their initial envelopes. The parallel dashed lines show the loci of identical mass-loss rates in the energy-limited evaporation regime, i.e., points along each line will have the same mass-loss rate.

planets are in the energy-limited, EUV-driven regime, and the color belts closely follow the parallel dashed lines.

Figure 6 shows the temporal evolution of the mass versus the semi-major axis $(a-M)$ distribution of the reference simulation (the XE simulation). The $a-M$ distribution shows typical sub-populations, such as the numerous low-mass "failed cores" that only accrete a limited quantity of gas, as well as many giant planets that preferentially form outside of the snow line (Ida \& Lin 2004; Mordasini et al. 2009). The color of each point in Figure 6] denotes the fraction of the initial envelope that is lost for this planet (i.e., 1.0 indicates that a planet has lost all of its initial $\mathrm{H} / \mathrm{He}$, and 0 means that the planet retains its entire envelope). The black region in the bottom right corner corresponds to the planets that have lost their entire initial envelope. The snapshot at 0.11 Gyr presents a greatly increased black bare-core region; however, the further increase is small for 1 and 5 Gyr. The critical planetary mass below which a planet can lose its entire envelope $\left(M_{\text {crit }}\right)$ has the form $M_{\text {crit }}(t)=M_{\text {crit }}\left(t, a_{0}=\right.$ $0.06 \mathrm{AU})\left(a / a_{0}\right)^{-1}$. For example, at $\mathrm{t}=10 \mathrm{Gyr}$ the critical planetary mass is $M_{\text {crit }}(a=0.06 \mathrm{AU}) \simeq 10 M_{\oplus}$, while
$M_{\text {crit }}(a=0.7 \mathrm{AU}) \simeq 1 M_{\oplus}$. Most of the fully depleted planets are low-mass "failed cores" $\left(M \leq 10 M_{\oplus}\right)$ that only accrete a small envelope during the formation stage. Although these planets have evaporated to bare, rocky cores, they do not lose a substantial amount of their total mass because their initial envelope masses are substantially lower than their core masses. Neptunian planets have greater initial envelope mass fractions, but it is difficult for them to lose a large portion of their envelopes. All of the Jovian planets retain most of their envelopes. Thus, the $a-M$ distribution of the entire planet population exhibits nearly no change: the four snapshots in Figure 6] all have similar shapes. Notably, herein we only include planets with $a \geq 0.06 \mathrm{AU}$; these results will differ for planets at very close-in orbits.

However, the $a-R$ distribution of the entire planetary population is clearly modified by evaporation. Figure 7 shows the $a-R$ distribution of the same reference simulation. First, certain features are related to the planet formation and evolution model. For example, the empty region from 0.2 to $2 \mathrm{AU}$ at $0.11 \mathrm{Gyr}$ is an artifact of using a minimal core mass of $1 M_{\oplus}$ in the formation cal- 

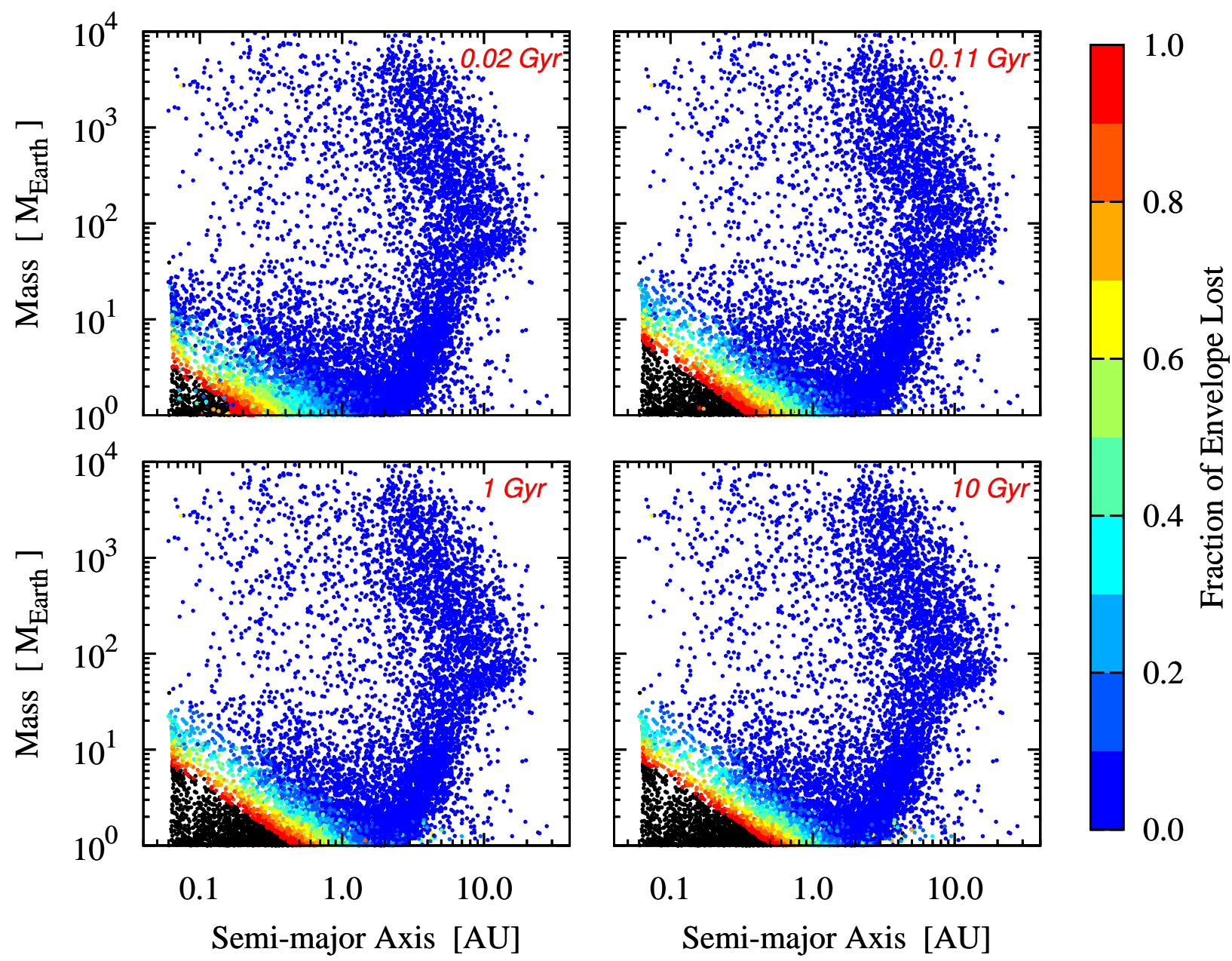

Semi-major Axis [AU]

FIG. 6. - The temporal evolution of the planetary mass and semi-major axis distribution of the reference simulation. The color of each point shows how much of the initial envelope was lost $\left(M_{\text {lost }} / M_{\text {initial }}\right)$. The black points are the planets that have lost all their initial envelopes. Note the large population of close-in, low-mass planets that have evaporated to bare cores.

culations. This region is left empty because protoplanets inside the ice line quickly accrete all planetesimals in their feeding zones. Therefore, their luminosities are low and are mostly attributed to gas accretion. For a fixed core mass under these circumstances, the envelope mass increases with orbital distance (see Ikoma \& Hori 2012) because more gas can be bound at lower nebula temperatures, which translates into a larger radius and makes the hollow higher at larger distances (to approximately $2 \mathrm{AU})$. Outside of this distance, another effect becomes dominant: the solid accretion timescale is longer, which makes that certain planets have high luminosities (due to planetesimal accretion) at the end of the disk lifetime. These planets can only hold tenuous envelopes and no longer retain a relationship with the orbital distance. In reality, there is no minimal core mass; therefore, this empty region should not exist in the actual $a-R$ plot. A very similar artifact can be seen in Fig. 7 of Owen \& Wu (2013). One real visible effect of planetary evolution can be observed by the decrease of the planetary radii of the entire population due to planet cooling. Note the sharp upper limit for the planetary radius, which is artificially sharp because, first, no special bloating mech- anisms are included (e.g., ohmic heating, Batygin et al. 2011), and second, the opacity, which can affect cooling (Vazan et al. 2013), is identical for each planet.

The features related to evaporation are shown by the color of each point, which represents the fraction of the initial envelope that evaporated. Here, we use black points to indicate the planets that have lost their entire envelopes (the bottom left region of each panel). As indicated, when a planet becomes a bare rocky core, it settles to the bottom of the $a-R$ plane and detaches from the planets that retain at least a portion of their initial envelopes; this settling leads to the formation of an empty region that runs diagonally downward in the $a-R$ plane between 0.06 and $0.5 \mathrm{AU}$. This empty diagonal belt separates the bare rocky cores from the planets that retain an envelope, which, henceforth, we refer to as an "evaporation valley". The evaporation valley occurs because the radius of a purely rocky planet is substantially smaller than that of a planet with both a core and gaseous envelope. For example, an envelope at only $0.1 \%$ of the planetary mass can dramatically enhance the planetary radius (Figure 2). Additionally, the last $0.1 \%$ of the envelope is lost on a short timescale, $\sim 10^{5} \mathrm{yrs}$; therefore, 

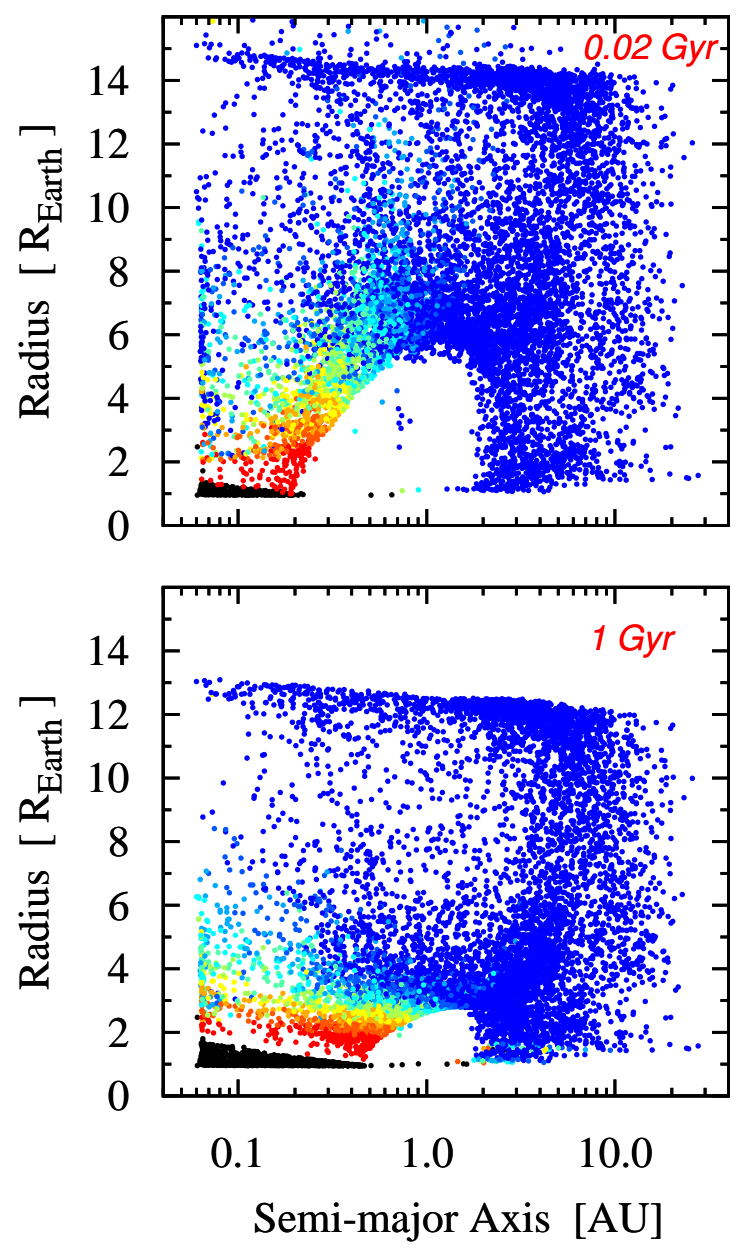
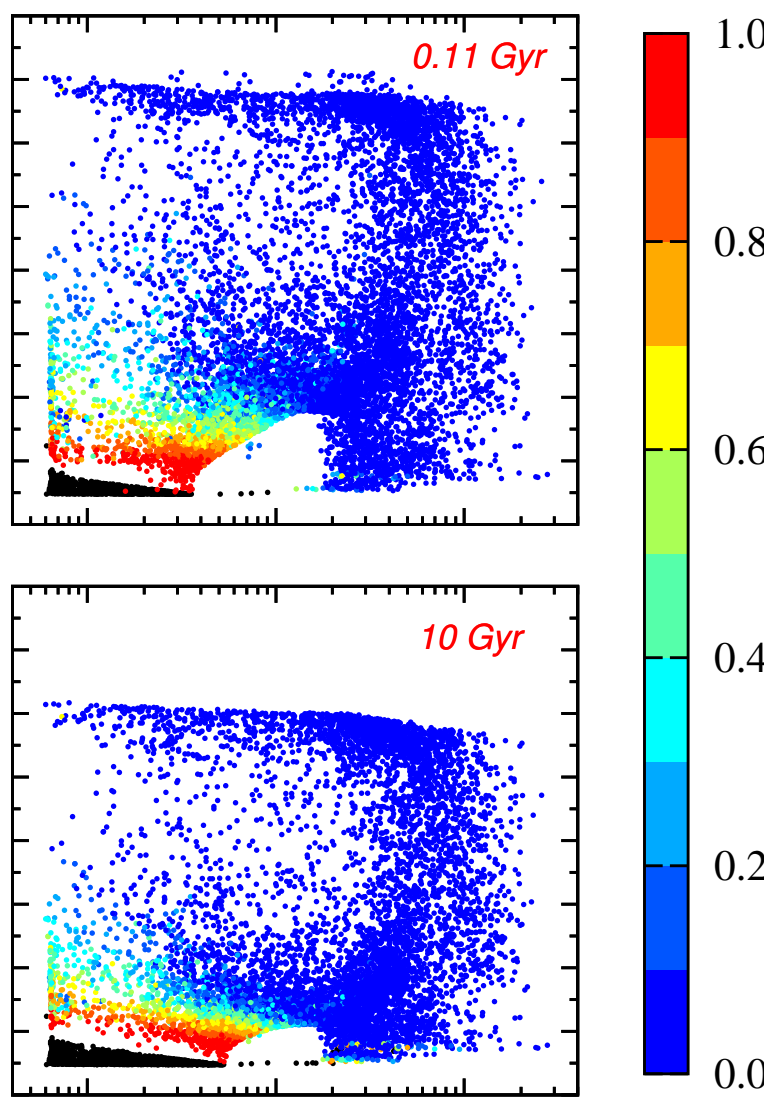

0.8

0.6 范

0.2

0.0
0.1
1.0
10.0
Semi-major Axis [AU]

FIG. 7.- Temporal evolution of the planetary radius and semi-major axis distribution of the reference simulation. The color of each point shows how much of the initial envelope was lost. The black points are the planets that have lost all their initial envelopes. Above the black points, a separating "evaporation valley" that runs diagonally downward from 0.06 to 0.5 AU at 5 Gyr is clearly visible. The empty region at intermediate orbital distances (extending at $0.02 \mathrm{Gyr}$ from 0.2 to $2 \mathrm{AU}$ ) is in contrast an artifact of assuming a minimal planetary mass of $1 M_{\oplus}$, and has no physical meaning. Note that all planets start with a primordial $\mathrm{H} / \mathrm{He}$ envelope. In reality, this is likely not the case for all low-mass planets.

we are unlikely to detect a planet when it lies in the evaporation valley. Thus, an empty valley appears in the $a-R$ plane after many low-mass planets have become bare, rocky cores. At $0.02 \mathrm{Gyr}$, the valley only appears within $\sim 0.1 \mathrm{AU}$, and rapidly extends to $\sim 0.3 \mathrm{AU}$ at $0.11 \mathrm{Gyr}$. Clearly, the empty valley is only expected if all low-mass planets begin with significant $\mathrm{H} / \mathrm{He}$ envelopes, which is unlikely. We discuss this topic further in $\S 5.1$. The separated distribution of low-mass planets within $0.5 \mathrm{AU}$ suggests a bimodal size distribution of the close-in lowmass planets, which was first theoretically observed by Owen \& Wu (2013). A similar but weaker structure was also demonstrated by Lopez \& Fortney (2013). We show the size distributions for our synthetic planet populations in $\oint 4.5$.

\subsection{Synthetic Planets: B, influence of Parameters}

To determine how our results depend on the evaporation description, we simulate the evolution of the same nominal planet population but using different evaporation models. Table 1 lists the details for these sim- ulations. Simulation XE is our reference. Simulation NoEV is planetary evolution without evaporation. Simulation SatE includes only EUV-driven evaporation and assumes that the stellar EUV emissions are saturated during the first 100 Myr of planetary evolution. Simulation XE2 uses the nominal evaporation model, but the heating efficiency in both the X-ray $(\epsilon=0.2)$ and energy-limited EUV regime $(\epsilon=0.12)$ are twice as high compared with the XE simulation. Simulation L12 uses the energy-limited model from Lopez et al. (2012), which uses the total flux between 1-1200 $\AA$ as the incoming energy and 0.1 as the heating efficiency. Simulation B04 uses the energy-limited model from Baraffe et al. (2004), which includes a different temporal evolution of the XUV emission from a sun-like star and adopts a 100\% heating efficiency. All the simulations are evolved for 10 Gyr.

Because we are more interested in the potentially observable influence of these different evaporation models, in Figure 8, we plot the mass-loss rates of the six simulations at 5 Gyr in the semi-major axis versus planetary mass $\times$ mean density $\left(M_{\mathrm{p}}^{2} / R_{\mathrm{p}}^{3}\right)$ plane. At $5 \mathrm{Gyr}$, only 

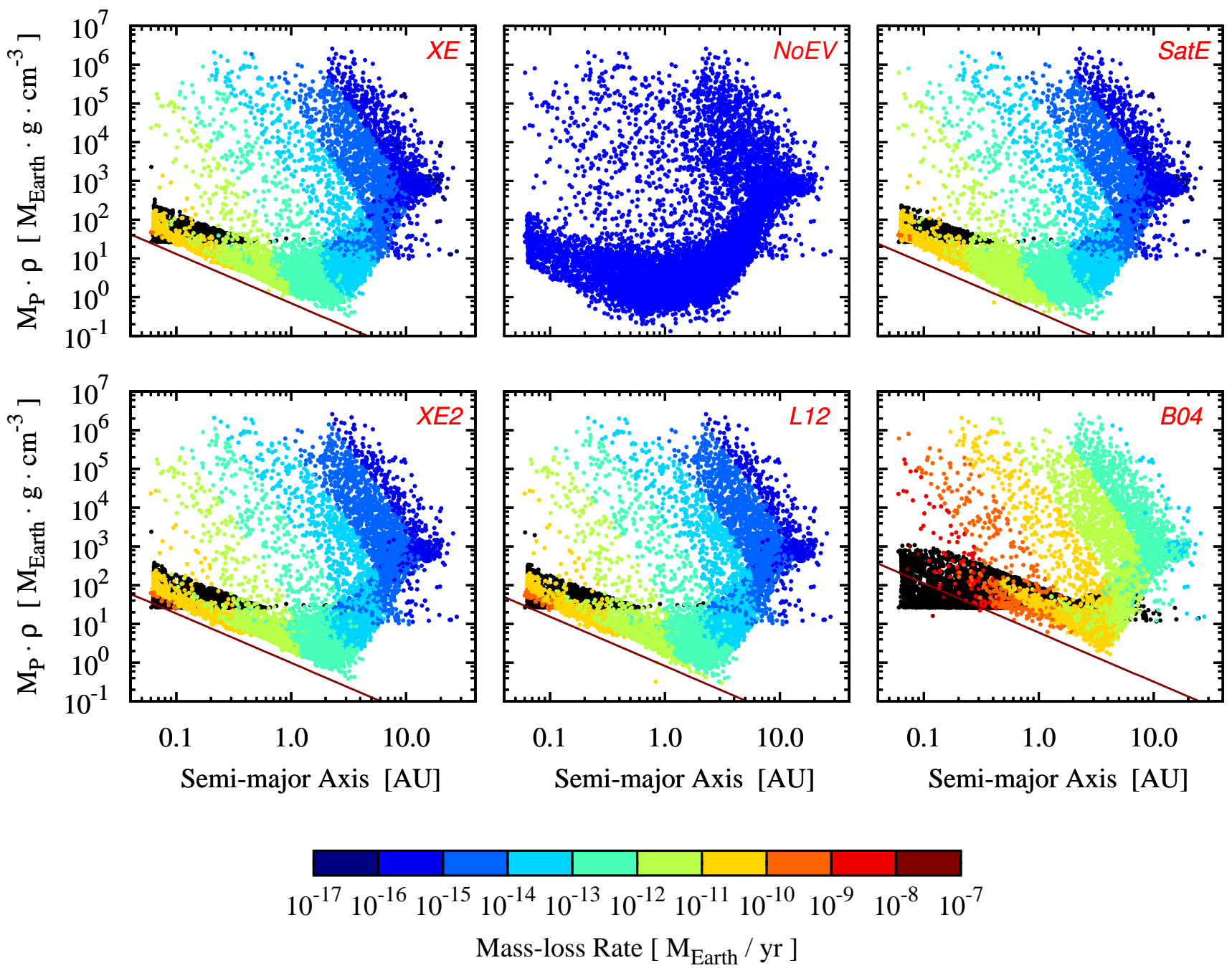

FIG. 8. - The mass-loss rates of the nominal planet population using different evaporation models (Table 1 ) at 5 Gyr in the $M_{\mathrm{p}} \cdot \bar{\rho}$ versus semi-major axis plane. The black points are the planets that have lost all their initial envelopes. The minimal planetary mass is $1 M_{\oplus}$. The solid line in each panel indicates the evaporation threshold.

Simulation B04 leads to planets with evaporation rates greater than $10^{-8} M_{\oplus} \mathrm{yr}^{-1}$. Simulation B04 is also the only simulation in which even a Jovian planet can evaporate to a rocky core. At $110 \mathrm{Myr}$, the largest mass-loss rates of the gas giants within 0.1 AU for Simulation B04 are $\sim 10^{-6} M_{\oplus} \mathrm{yr}^{-1}$ (not shown in the plot), which indicates that these planets lose at least $100 M_{\oplus}$ in the first $100 \mathrm{Myr}$ of planetary evolution because the massloss rate of a planet decreases with time. Thus, many planets in Simulation B04, including certain close-in Jovian planets, eventually lose their entire envelope. The mass-loss rates for the other four simulations are significantly smaller; the typical rate for a planet within 0.1 AU at 5 Gyr is $\sim 10^{-10} M_{\oplus} \mathrm{yr}^{-1}$ and is $\sim 10^{-8} M_{\oplus}$ $\mathrm{yr}^{-1}$ at $110 \mathrm{Myr}$ (not shown in the plot).

The evaporation timescale $M_{\mathrm{p}} / \dot{M}$ is proportional to $M_{\mathrm{p}}^{2} /\left(R_{\mathrm{p}}^{3} F\right)$ ( $F$ is the incoming flux) in an energy-limited regime; thus, an evaporation threshold is expected in the semi-major axis versus planetary mass $\times$ the mean density $\left(M_{\mathrm{b}} \cdot \bar{\rho}\right)$ plane (Jackson et al. 2012; Lopez et al. 2012; Owen \& Wu 2013). Low-mass, low-density planets below this threshold at the beginning of planetary evolution will be evaporated to bare, rocky cores at high mean densities, and eventually, no planets with $\mathrm{H} / \mathrm{He}$ are below the threshold, as demonstrated by the solid lines and black dots in Figure 8. A similar threshold is also apparent in the NoEV simulation, which does not include evaporation; however, in this instance, the cut-off is unclear (as in the evaporation-inclusive simulations), and the threshold is at the lower value of $M_{\mathrm{p}} \cdot \bar{\rho}$. This lower limit is an artifact of using a the minimal planetary mass of $1 M_{\oplus}$, which, indicates that the envelope mass for a fixed core mass increases with orbital distance, as discussed above. Evaporation renders this threshold clearer and raises it in the $M_{\mathrm{p}} \cdot \bar{\rho}$ plane. With a substantially stronger evaporation model, the evaporation threshold is so high that it intersects the bare, rocky cores; hence, the evaporation threshold becomes blurred, as demonstrated in the B04 simulation. If planets with masses lower than $1 M_{\oplus}$ are included in our synthetic population, then the evaporation threshold can also be blurred because the lowmass bare rocky cores would extend to lower values of $M_{\mathrm{p}} \cdot \bar{\rho}$ below the threshold. Examples of such low-mass 

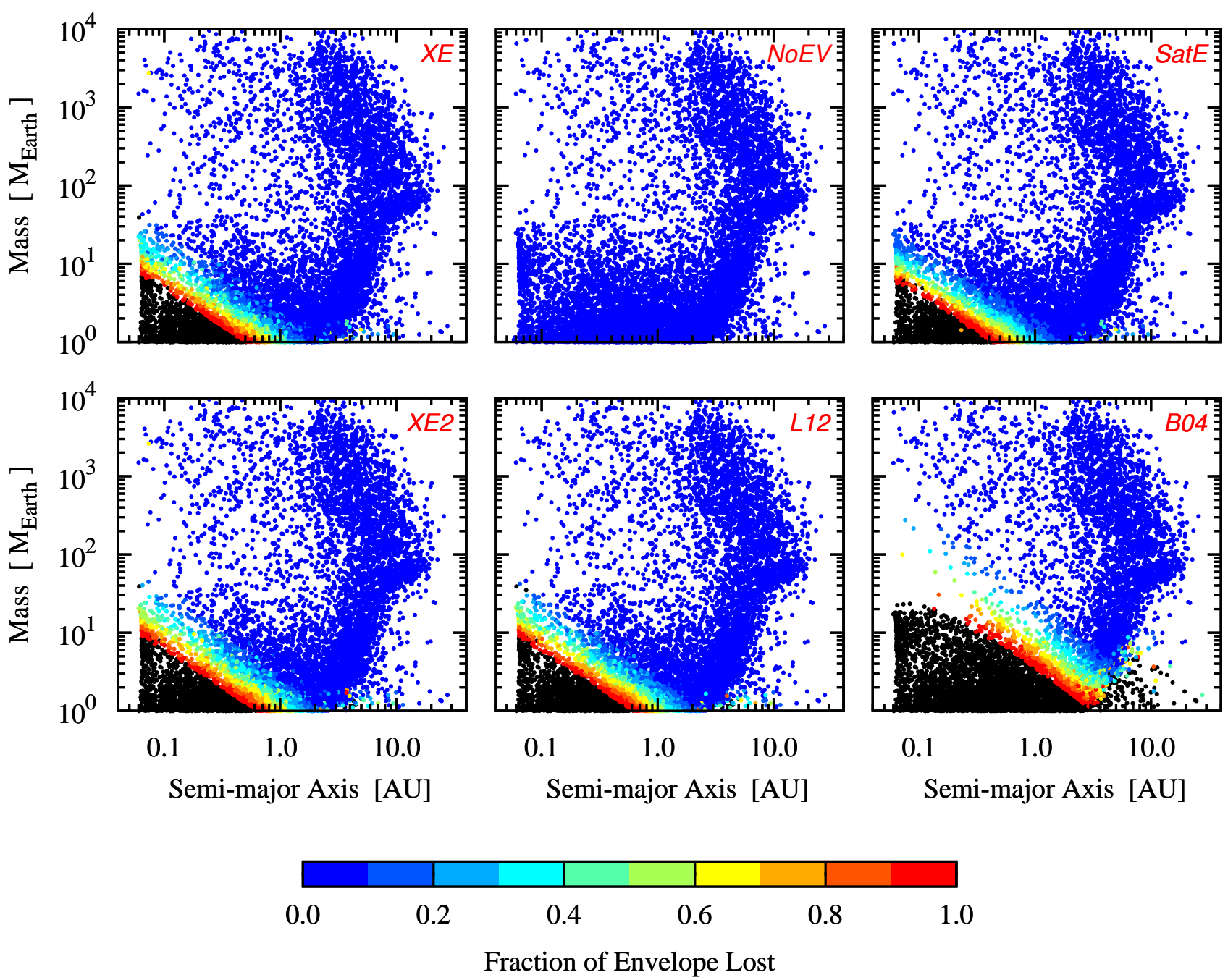

FIG. 9.- The planetary mass versus semi-major axis distributions of the nominal planet population using different evaporation models at 5 Gyr (Table 1). The color of each point shows how much of the initial envelope was lost. The black points are the planets that have lost all their initial envelopes.

cores include Kepler-10b and Corot-6b (see Figure 6 in Lopez et al. (2012)).

Figure 9 shows the $a-M$ distributions at 5 Gyr. With the exception of Simulation B04, the remaining five simulations present similar $a-M$ distributions. The difference for B04 is the severe depletion of planets with masses at 30-50 $M_{\oplus}$ within 0.2 AU. At distances smaller than those modeled here $(a \lesssim 0.03 \mathrm{AU})$, the observed planet population includes a desert that may be related to evaporation (Beaugé \& Nesvorný 2013; Kurokawa \& Nakamoto 2014). In each simulation, the black bottom left corner corresponds to the planets that have lost their entire envelope. Although the heating efficiency in XE2 is twice that of XE, the black corner in XE2 does not show a large difference compared with XE. These data indicate that long-term evolution of the entire planet population is not extremely sensitive to the heating efficiency of the evaporation model, at least within the framework of the models listed here. However, if a substantially more violent evaporation model is used, as for B04, the $a-M$ distribution of the entire planet population noticeably changes.
Figure 10 shows the $a-R$ distributions of the six simulations at $10 \mathrm{Gyr}$. The color of each point indicates the ratio of the envelope mass to the core mass, $f_{\text {env/core }}$. Here, all of the simulations that include evaporation show a distinct feature compared with NoEV: an evaporation valley in the radius distribution at $\sim 2 R_{\oplus}$. The $f_{\text {env } / \text { core }}$ of each planet does not change in the NoEV simulation; thus, based on the colors in the NoEV snapshot, the initial $f_{\text {env/core }}$ of a planet generally scales with its core mass. This finding is expected based on the long KelvinHelmholtz timescales for gas accretion of low-mass cores. For example, only the planets within $0.1 \mathrm{AU}$ with a core larger than $10 M_{\oplus}$ can have a $f_{\text {env/core }}$ that exceeds $80 \%$; low-mass planets with a core smaller than $2 M_{\oplus}$ typically have a $f_{\text {env/core }}$ that is less than $10 \%$. Thus, most low-mass planets with cores at $1-3 M_{\oplus}$ can be quickly evaporated to bare, rocky cores after evolution has begun (even in simulation SatE, which includes the weakest evaporation model). However, this clear valley only occurs if all these low-mass planets begin with a primordial $\mathrm{H} / \mathrm{He}$ envelope, which is unlikely in reality. In the actual formation process, some low-mass planets may reach 

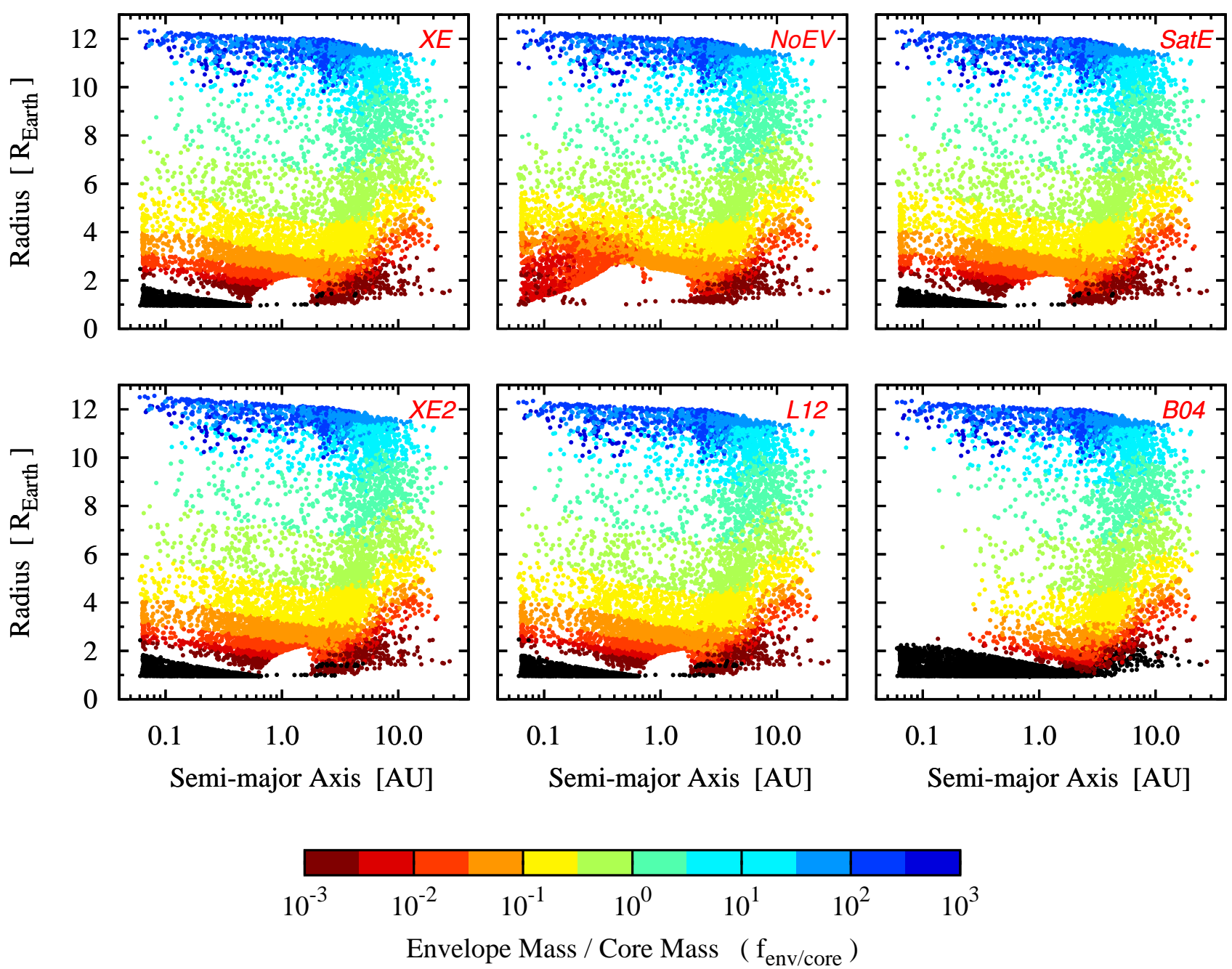

FIG. 10.- The planetary radius versus semi-major axis distributions of the nominal planet population using different evaporation models at $10 \mathrm{Gyr}$ (Table 1). The color of each point shows the ratio of the planetary envelope mass to the core mass, $f_{\text {env } / \text { core }}$. The black points are the planets that have lost all their initial envelopes. It is obvious that the simulation B04 is incompatible with the observed $a-R$ relationship.

their final mass only after the dissipation of the gaseous nebula, leading to planets without significant primordial $\mathrm{H} / \mathrm{He}$ envelopes. This is in contrast with our synthetic population where no growth via giant impact is included after the protoplanetary disk has dissipated. When the low-mass planets become bare, rocky cores, they settle to the bottom of the $a-R$ panel and are separated from the planets that retain some $\mathrm{H} / \mathrm{He}$. The evaporation valley becomes a large void region for B04, for which the mass-loss rates are so high that most planets within 0.2 AU have evaporated to bare cores, including certain gas giants with $M_{\mathrm{p}} \lesssim 400 M_{\oplus}$. Such strong planet depletion between $\sim 2$ and $\sim 10 R_{\oplus}$ is not presented in the observed data (including Kepler candidates), which implies that an 100\%-efficient, energy-limited evaporation model is incompatible with the observed radius distribution of the extrasolar planets.

\subsection{Mass-Radius Relationship of Close-in Planets}

Figure 11 compares the mass-radius relationship of the planets between 0.06-1 AU at 5 Gyr for the six simula- tions using different evaporation models. The planetary radii plotted in the figure are at the optical depth $\tau=$ $2 / 3$. Compared with the old grey model (Mordasini et al. 2012a), the semi-grey model used for the atmosphere increases the radius of close-in planets due to stellar irradiation (Guillot 2010). This effect is demonstrated by the low-mass planets at small semi-major axes, such as planets with masses of $1-2 M_{\oplus}$ but radii of $3-5 R_{\oplus}$ in the simulation NoEV, which does not include an evaporation model. These low-mass planets have low densities. For example, the mean density of a $1.5 M_{\oplus}, 4 R_{\oplus}$ planet is only $\sim 0.13 \mathrm{~g} \mathrm{~cm}^{-3}$.

The evaporation valley in Figure 10 and evaporation threshold in Figure 8 can also be observed in the massradius relationships in Figure 11. The black bar at the bottom of each panel in the five simulations that included evaporation corresponds to the rocky cores of the planets that have lost their entire envelopes. Note that with the migration model used here, all close-in low-mass planets that become bare cores have only accreted inside of the iceline, giving them a rocky interior. This could be 

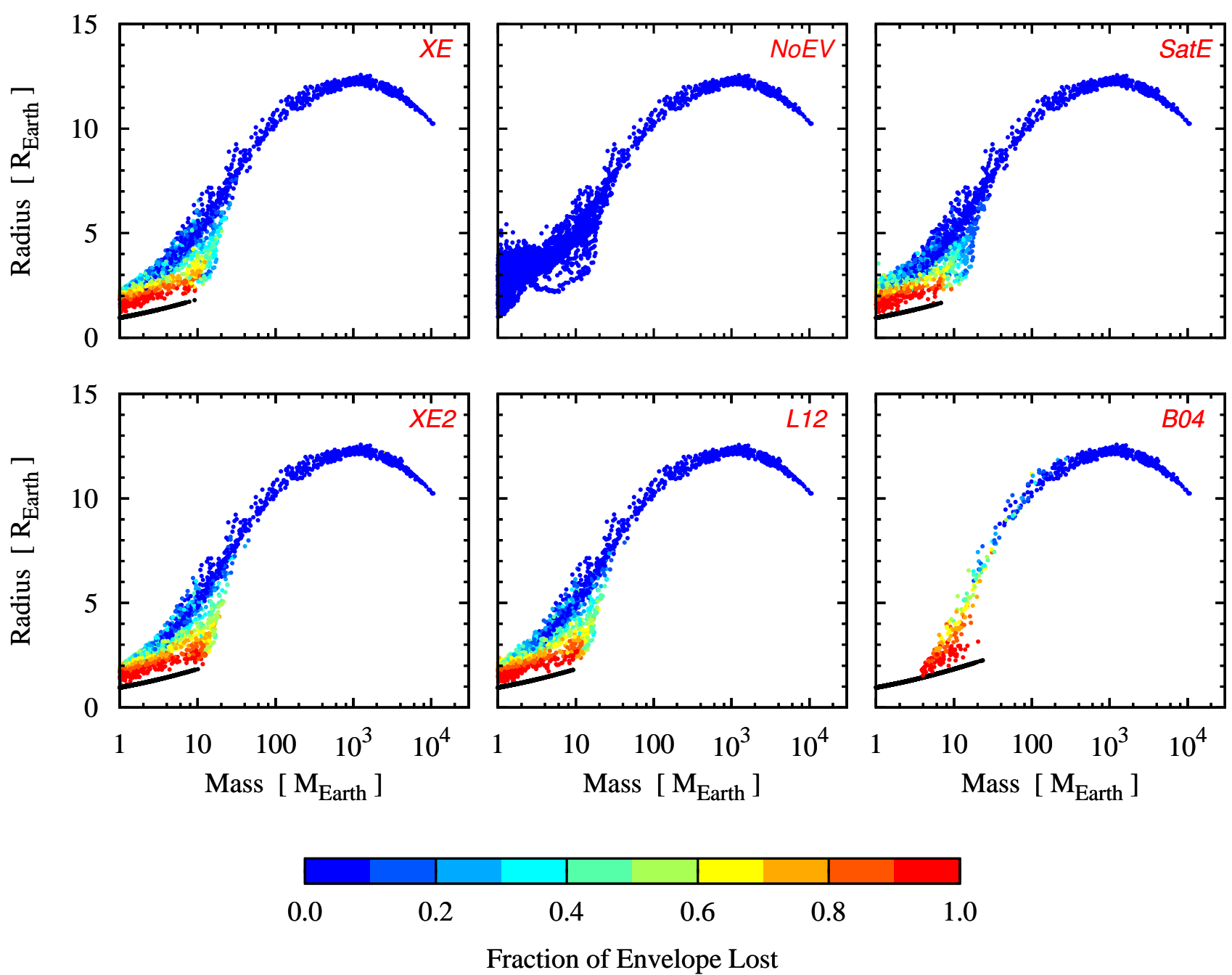

FIG. 11. - The mass-radius relationship of the planets between 0.06 and 1 AU at 5 Gyr for different simulations (Table 1 . The color of each point shows how much of the initial envelope was lost. The black points are planets that have lost all their initial envelopes. All the planets have an identical, solar composition opacity, a pure H/He envelope, and no bloating mechanisms are included. This means that the vertical width of the mass-radius relation is likely underestimated. The mass of the host star is $1 M_{\odot}$. All rocky planetary cores have a terrestrial composition (2:1 silicate-iron ratio).

different for higher migration rates, or if several planets form concurrently in one disk (Alibert et al. 2013). In the current model, we assume that all rocky cores have an identical composition (2:1 silicate:iron ratio, as in Earth). In reality, this ratio (and the composition of refractory element) depends on the stellar composition and a planet's formation history, such as large impacts. The black bar creates a gap at $\sim 2 R_{\oplus}$, which separates the bare cores from the planets that retain at least a portion of their initial envelopes. This feature clearly corresponds to the evaporation valley in Figure 10). The length of this black bar is related to the efficiency of evaporation mechanism. Simulation SatE includes the lowest mass-loss rates; consequently, it produces the shortest black bar. Simulation B04 produces the longest black bar, extending to $\sim 20 M_{\oplus}$, and certain black points are massive cores from stripped gas giants. In Simulation B04, no planets with $M_{\mathrm{p}} \lesssim 5 M_{\oplus}$ within 1 $\mathrm{AU}$ maintain their primordial $\mathrm{H} / \mathrm{He}$. Another difference between NoEV and the other five simulations is the dis- appearance of low-mass, very low-density planets. This result is similar to the evaporation threshold in Figure 8. When evaporation is included in planetary evolution, the bloated envelopes of close-in, low-mass planets are rapidly removed. Thus, an upper threshold is depicted in the bottom left corner of the mass-radius distribution (for planets within $1 \mathrm{AU}$ ). Planets that are initially above this threshold will lose at least a portion of their initial envelopes until they are sufficiently dense that they lie below the threshold. This threshold is also related to the efficiency of evaporation mechanism; in Simulation B04, the threshold occurs at larger planetary masses compared with Simulation SatE.

\subsection{Synthetic Planets: $C$, non-isothermal Migration and Different Grain Opacities}

Various evaporation models have been applied to the nominal planet population that is calculated using the isothermal type I migration rate reduced by a factor of 0.1 Tanaka et al. (2002). This is an artificial fac- 

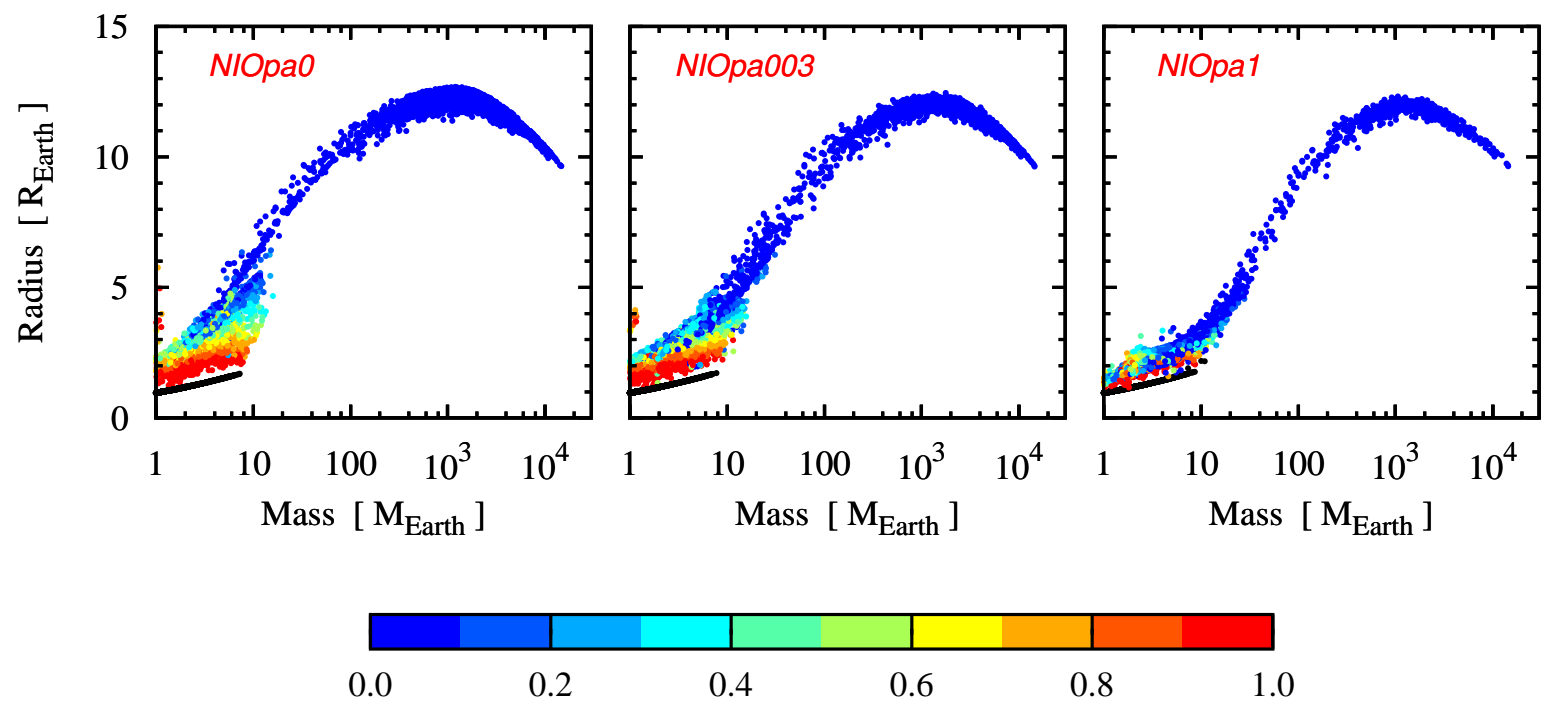

Fraction of Envelope Lost

FIG. 12. - The mass-radius relationship of the planets between 0.06 and 1 AU at 5 Gyr of the three planetary populations calculated using non-isothermal type I migration rates and different grain opacities (Table 1). The color of each point shows how much of the initial envelope was lost. The black points show the planets that have lost all their envelopes. All of the planets orbit around a sun-like star.

tor that prevents most synthetic planets from falling into the star (Benz et al. 2014). Recent studies have shown that, depending on the temperature profile of the disc, type I migration can also induce outward migration (e.g., Masset et al. 2006; Paardekooper \& Mellema 2008; Klev et al. 2009; Uribe et al. 2011). Thus, in principle, the artificial reduction factor may be eliminated even if the migration still seems to be too rapidly inward, mainly due to saturation of the corrotation torques (Benz et al. 2014). Here, we apply the nominal evaporation model to three synthetic populations that are all calculated using the full non-isothermal type I migration rate from Dittkrist et al. (2014). The difference between these three populations is the ISM grain opacity reduction factor, $f_{\text {opa }}$, which is used during the formation stage. For the planet population in Simulation NIOpa003, we use an $f_{\text {opa }}$ at 0.003 (the nominal value) during formation; in Simulation NIOpa0, the $f_{\text {opa }}$ equals 0 (no grain opacity), and in Simulation NIOpa1, the $f_{\text {opa }}$ equals 1 (full ISM grain opacity). The details are in Table 1 and Mordasini et al. (2014a). Lower grain opacity during formation phase yields a higher envelope mass for a given core mass because it is more efficient to radiate away the liberated potential energy, allowing the envelope to contract. This means that, at low $f_{\text {opa }}$, planets with a lower mean density are formed, which is indicated by a larger maximal radius for a given mass in the massradius diagram. In contrast, the atmospheric opacity during planetary evolution is identical for all three populations and is given via the opacity of a grain-free gas with a solar composition (Freedman et al. 2008).

The features that are related to evaporation, such as the black bottom left corner in the $a-M$ diagram, the evaporation valley in the $a-R$ distribution, and the black bar, which indicates purely rocky cores in the massradius plot, have been detailed above and are similar among these three populations. Here, we focus on the features related to the effect of $f_{\text {opa }}$. Figure 12 shows the mass-radius relationships of the planets within 1 AU for the NIOpa003, NIOpa0, and NIOpa1 simulations. One of the effects of the different grain opacities during formation is the number of giant planets (Mordasini et al. 2014a). As shown in the figure, the NIOpa0 group includes the most gas giants, while the NIOpa1 group shows the opposite result. This effect is more clearly demonstrated by the histogram of planet size distributions in Figure 14 in the following subsection. Another effect is that, in Simulation NIOpa0, the largest overall radius (at a mass of $\sim 4 M_{\text {Jupiter }}$ ) is slightly larger than for the other two simulations. At $f_{\text {opa }}=0$, even very low-mass cores can become supercritical and trigger giant planet formation (Movshovitz et al. 2010; Mordasini et al. 2014a). These giant planets with lowmass cores yield large planetary radii. A third effect, as extensively discussed in Mordasini et al. (2014a), is the effect of $f_{\text {opa }}$ on the mass-radius relationship of lowmass planets. With a low $f_{\text {opa }}$, even cores with only a few $M_{\oplus}$ can accrete significant envelope masses, thereby producing large radii. This result is clearly visible in Figure 12. For example, at $10 M_{\oplus}$, the maximum radii are approximately $3.5,5.5$, and $7.5 R_{\oplus}$ for $f_{\text {opa }}=1,0.03$, and 0 , respectively. The effect of the grain opacity during formation on the observable mass-radius relationship as discussed in Mordasini et al. (2014a) (who neglected evaporation) is also found here, even if the very low-mass, low-density planets are removed by evaporation.

\subsection{Comparisons with Kepler Candidates}

Figure 13 shows the temporal evolution of the normalized size distribution for Simulation XE. For comparison with the observations, we only show the size distributions for the planets within $1 \mathrm{AU}$. There are three peaks in the planet size distributions, all of which form at an early evolutionary stage. The first peak is at $\sim 1$ $R_{\oplus}$, which corresponds to the bare, rocky cores of the low-mass planets that have entirely lost their initial en- 

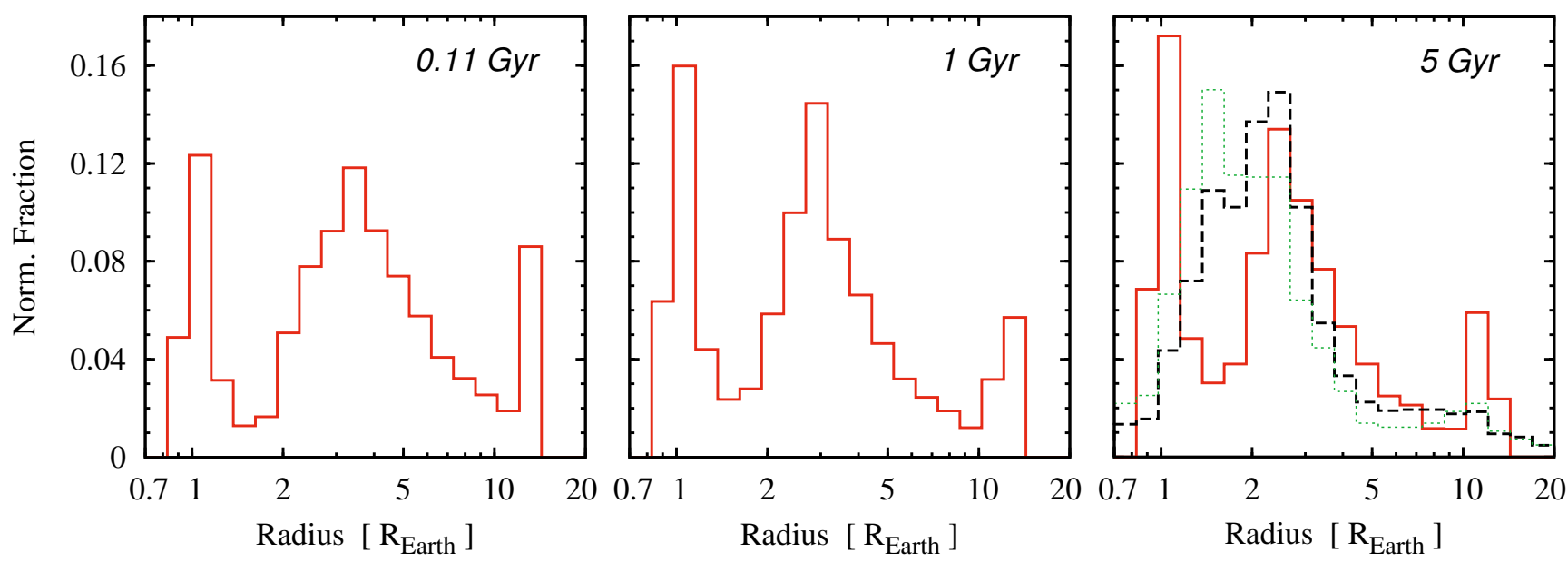

FIG. 13. - The normalized planet size distribution of the XE simulation at $0.1,1$, and 5 Gyr. The red solid line corresponds to the sub-population of the planets within $1 \mathrm{AU}$. In the $5 \mathrm{Gyr}$ panel, the black dashed line shows the size distribution of all Kepler candidates, while the green doted line shows the size distribution of Kepler candidates within 0.1 AU. Note that at radii $\lesssim 2 R_{\oplus}$, the Kepler data is affected by observational bias, and the size distribution of Kepler candidates with correction for survey completeness is a plateau at 1-3 $R_{\oplus}$ (Dong \& Zhu 2013; Petigura et al. 2013a).

velopes. The third peak, at $11-12 R_{\oplus} \sim 1 R_{\text {Jupiter }}$, indicates the sub-population of Jovian planets and is a consequence of degeneracy of the electrons in the interior; all giant planets with masses larger than $\sim$ Saturn have the same radius of approximately $\sim 1 R_{\text {Jupiter }}$. Compared with the actual population, this effect must be overestimated in our results because all planets evolved with the same opacity, and bloating mechanisms are not included. The middle peak at $2-4 R_{\oplus}$ corresponds to the sub-population of super-Earths and Neptunian planets that retain an envelope. Owen \& Wu (2013) found that evaporation leads to a bimodal distribution in planetary size with a planet deficit at approximately $2 R_{\oplus}$. Our results show a similar bimodal distribution with a minimum at approximately $2 R_{\oplus}$; however, this deficit is substantially more severe at $\sim 1.2-2 R_{\oplus}$.

The normalized size distribution of all Kepler candidates (released Feb 26, 2012), most of which are within 1 AU, are also plotted for comparison in the 5 Gyr panel. Notably, the Kepler data are biased and incomplete for $R \lesssim 2 R \oplus$ because the detection efficiency of the star and the detection sensitivity of the planet decrease towards small planetary radii and larger semi-major axes. The planetary occurrence of Kepler candidates with a correction for observational bias produces a nearly flat distribution at 1-3 $R_{\oplus}$ (Howard et al. 2012; Dong \& Zhu 2013; Petigura et al. 2013a, b). Figure 13 shows that the wide evaporation valley in our result is not compatible with Kepler data. There are three possible reasons for this observation. (1) Our evaporation model either overestimates evaporation or is too deterministic because all stars in our simulation have the same mass and XUV flux as a function of time. (2) We assume that all lowmass planets begin with a primordial $\mathrm{H} / \mathrm{He}$ envelope. In reality, there is likely a population of close-in, low-mass planets that formed without $\mathrm{H} / \mathrm{He}$ via planet impacts after the dissipation of the nebula (Terquem \& Papaloizou 2007; Ormel et al. 2012). (3) The distinct evaporation valley is related to our identical core composition $(2: 1$ silicate:iron ratio). As mentioned, the migration model (0.1 isothermal migration rate of Tanaka et al. 2002) predicts that all close-in low-mass planets loosing the entire envelope have a rocky interior (migration only inside of the iceline). In reality, the actual core composition of close-in planets might be highly diverse (i.e., contains some ice). In the 5 Gyr panel, we also plotted the normalized size distribution for Kepler candidates within $0.1 \mathrm{AU}$, which are planets that are more likely to be eroded by evaporation. These candidates accumulate at $\sim 1-3 R_{\oplus}$, while the close-in Kepler candidates corrected for the observational bias have a nearly flat distribution at $1-3 R_{\oplus}$ (Dong \& Zhu 2013; Petigura et al. 2013a, b). Thus, the evaporation valley in our synthetic planet populations was not observed in the Kepler data. In a following paper, we show that, by varying the ice fraction of the planetary cores, the radii of the bare, rocky cores can be substantially larger; the dip in the evaporation valley is thus eliminated. This means that important observational constrains for planet formation and migration theory can be deduced from evolutionary models with atmospheric escape (cf. Lopez \& Fortnev 2013).

Figure 14 compares the normalized size distribution of Kepler candidates with our non-nominal simulations. With the exception of NoEV, which does not include evaporation, and B04, which has a 100\% heating efficiency in the energy-limited regime, the remaining seven simulations show a bimodal distribution at small sizes. As mentioned, an additional peak is at $1 R_{\text {Jupiter }}$ for the gas giants. Simulation NoEV yields only one peak at $3-4 R_{\oplus}$; this occurs because the low-mass planets maintain their envelopes and, hence, have large planetary radii. The simulation B04 group is another extreme case that yields a single peak at $1-2 R_{\oplus}$. Most of the low-mass planets in Simulation B04 evaporated to bare, rocky cores; thus, the number of Neptunian planets that retain an envelope is too small to produce a second peak. In the other seven simulations, the inner peak in the bimodal distribution is at 1.0-1.2 $R_{\oplus}$, which corresponds to bare, rocky cores with a $2: 1$ silicate:iron ratio. For different simulations, the outer peaks in the bimodal dis- 

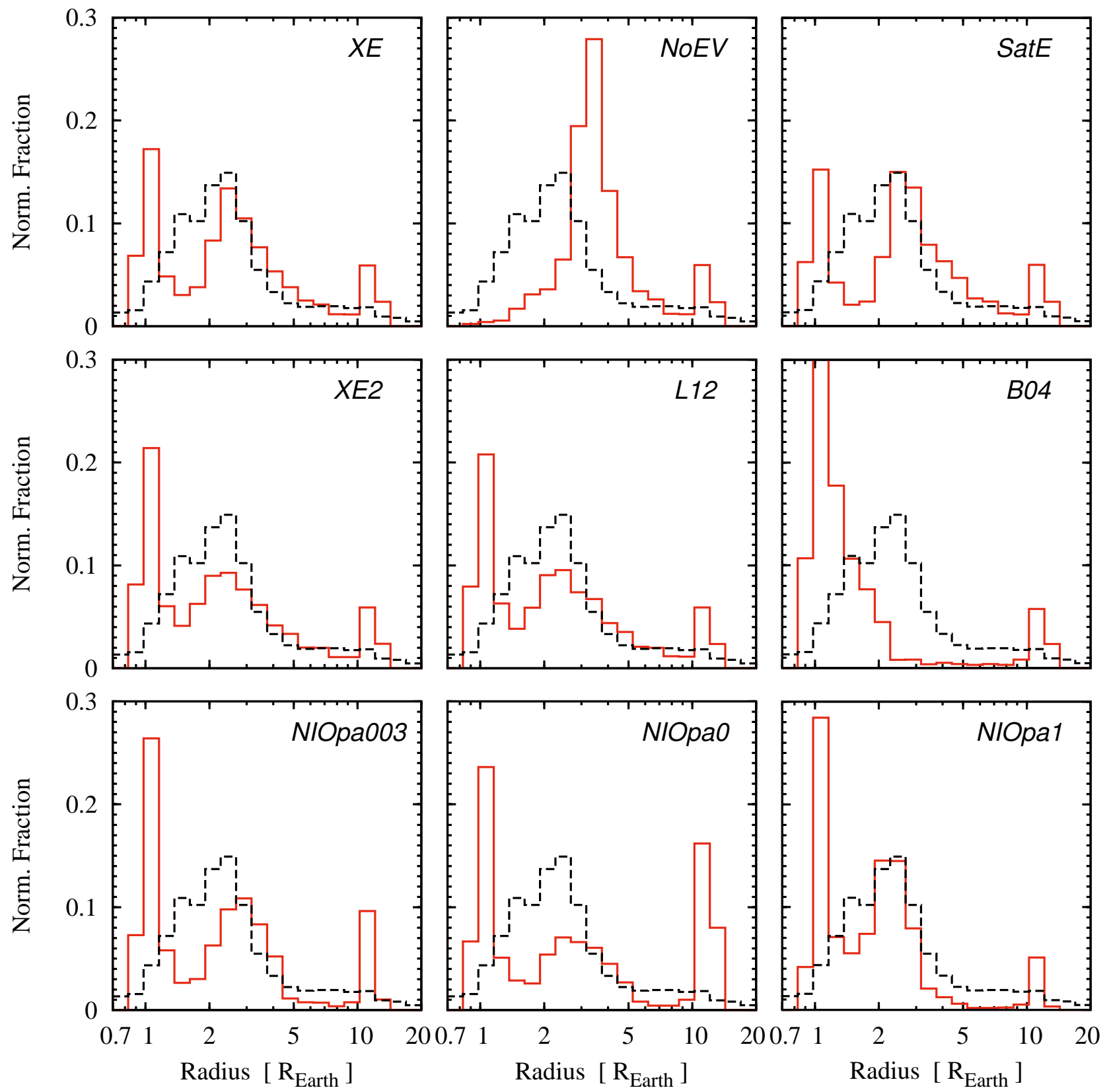

FIG. 14.- The normalized planet size distributions of all the simulations at 5 Gyr (Table 1). In each panel, the red solid line shows the synthetic population with $0.06<a / \mathrm{AU}<1$, and the black dash line shows size distribution of all Kepler candidates.

tribution differ slightly in both magnitude and location. A stronger evaporation model will produce a lower outer peak and move the position of the peak to smaller planet sizes, as shown in the top two rows of Figure 14, where the only difference between these simulations is the evaporation model. The effect of the grain opacity reduction factor, $f_{\text {opa }}$, is apparent in the bottom row of Figure 14. Because planets grow faster at smaller $f_{\text {opa }}$, the NIOpa0 group contains the largest number of gas giants. Consequently, the NIOpa0 group has the highest peak at $\sim 12$ $R_{\oplus}$; the NIOpa1 group is the opposite.

\section{DISCUSSION}

\subsection{The Bimodal Distribution and Evaporation Valley}

The bimodal distribution for planet sizes at approximately $2 R_{\oplus}$ was first observed by Owen \& Wu (2013), in which the hydrodynamic evaporation of a theoretical planet population was studied. Lopez \& Fortney (2013) observe a diagonal band, on which planets are relatively rare; the bimodal distribution near $2 R_{\oplus}$ is less clear in their results. In our results, this diagonal band Lopez \& Fortney (2013) becomes a distinct evaporation valley. This evaporation valley separates the bare, rocky cores from the planets that retain an envelope (Figure 10). The valley is $\sim 0.5 R_{\oplus}$ wide and occurs at different planet sizes (from $\sim 1$ to $\sim 2.5 R_{\oplus}$ ) depending on the semi-major axis.

We find that such empty valleys are closely related 

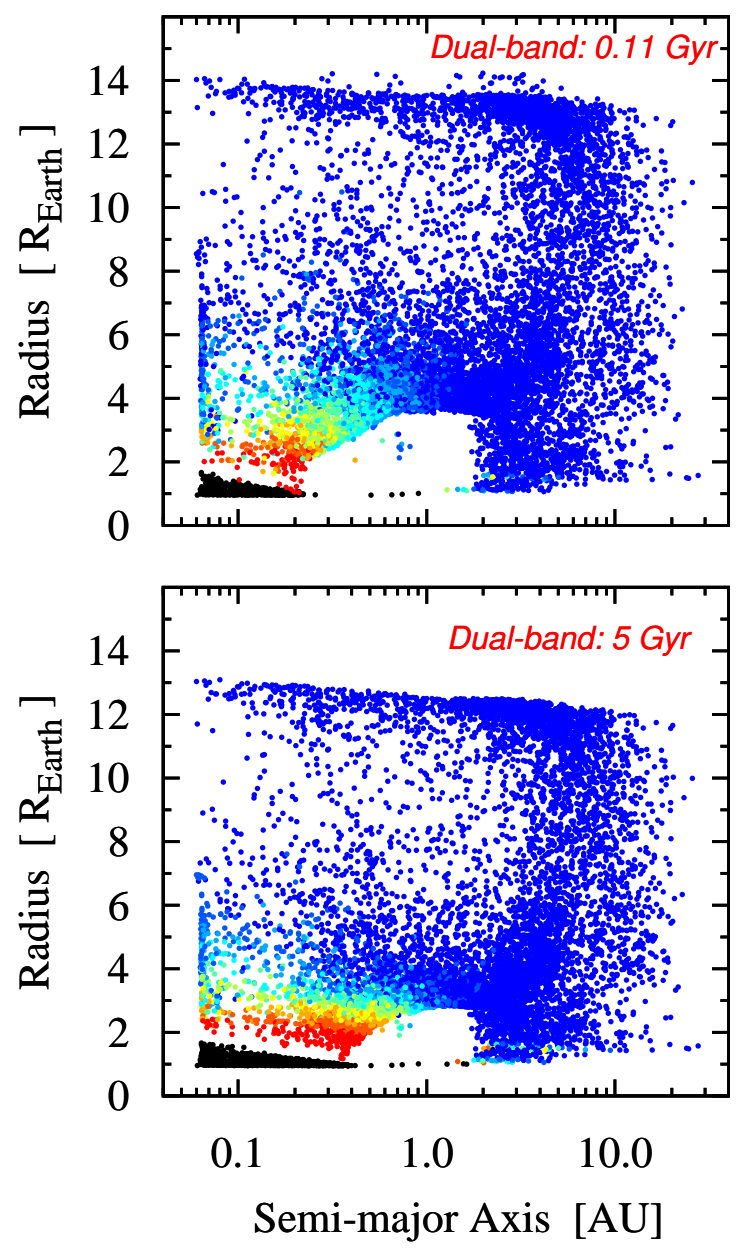
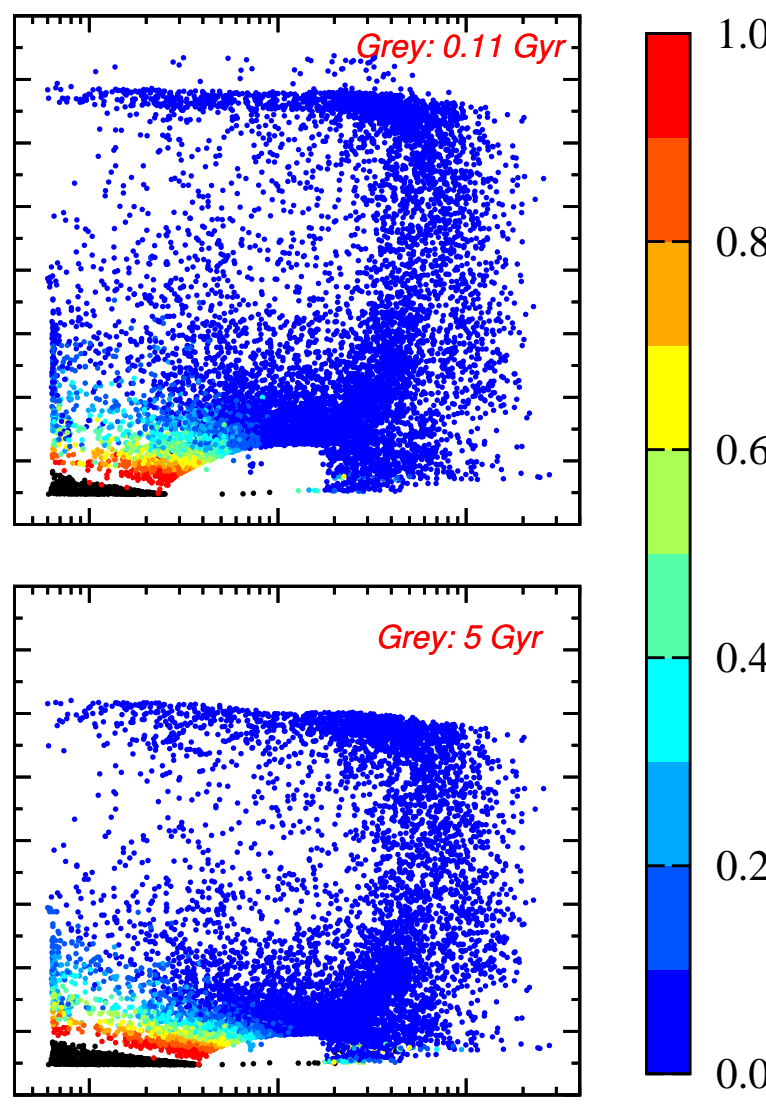

1.0

0.8

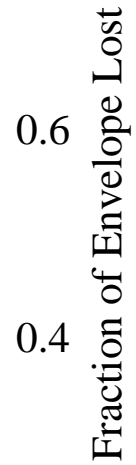

0.2

0.0
0.1
1.0
10.0
Semi-major Axis [AU]

FIG. 15. - The $a-R$ distributions of the XE and GREY simulations at 0.11 and 5 Gyr. The color of each point shows how much of the initial envelope was lost. The black points are the planets that have lost all their initial envelopes. The planetary radii are smaller and the evaporation valley is also narrower in the GREY simulation, but the general, population-wide impact of evaporation is similar in the two simulations.

to the initial characteristics of the synthetic planet population. In our synthetic planet population, all close-in planets begin with a primordial $\mathrm{H} / \mathrm{He}$ envelope, and their rocky cores have a 2:1 silicate:iron ratio. Due to the long Kelvin-Helmholtz timescales of low-mass cores, low-mass planets can only have a small initial $f_{\text {env/core, which is }}$ proportional to the planetary core mass. As shown in the NoEV simulation snapshot in Figure 10. low-mass planets within $0.5 \mathrm{AU}$ with a radius $<4 R_{\oplus}$ typically include a $f_{\text {env } / \text { core }}<10 \%$. Only a planet with $>6 R_{\oplus}$ can have a $f_{\text {env } / \text { core }} \geq 80 \%$. Thus, low-mass planets are vulnerable to evaporation to bare rocky cores due to their small envelopes and small gravitational binding energies; these planets form a peak at about $1 R_{\oplus}$ in the bimodal radius distribution of low-mass planets (trimodal, if the giants are included). Most close-in Neptunian planets can keep at least a portion of their initial envelopes at the end of evolution; they form the second peak at about $2-3 R_{\oplus}$ in the bimodal distribution. In a forthcoming paper, we show that the dip of the evaporation valley can be removed by varying the ice fractions of planetary cores: the sizes of bare low-mass icy cores can be $\sim 2 R_{\oplus}$.

\subsection{Insensitivity to the Boundary Conditions}

Although the semi-grey model is a significant improvement over the grey model used in Mordasini et al. (2012a), it does not include the effects of nongrey thermal opacities (Parmentier \& Guillot 2014; Parmentier et al. 2013b). Neglecting non-grey effects in the planetary atmosphere boundary may lead to an inaccurate planetary radius and, hence, mass-loss rates. Fortunately, we find that the final radius distribution of the entire planet population is not very sensitive to the outer boundary condition. The exact planetary radius and mass-loss rate are clearly important for individual planets but not so much for the overall statistical impact on the planet population.

To clarify this, we perform a comparison numerical experiment, the GREY simulation, using our nominal planet population but with the previous grey atmospheric model from Mordasini et al. (2012a). The grey model assumes that stellar irradiation is absorbed at the upper atmosphere where optical depth equals $2 / 3$. For the GREY simulation, we also use the nominal evaporation model (i.e., both X-ray- and EUV-driven evapora- 


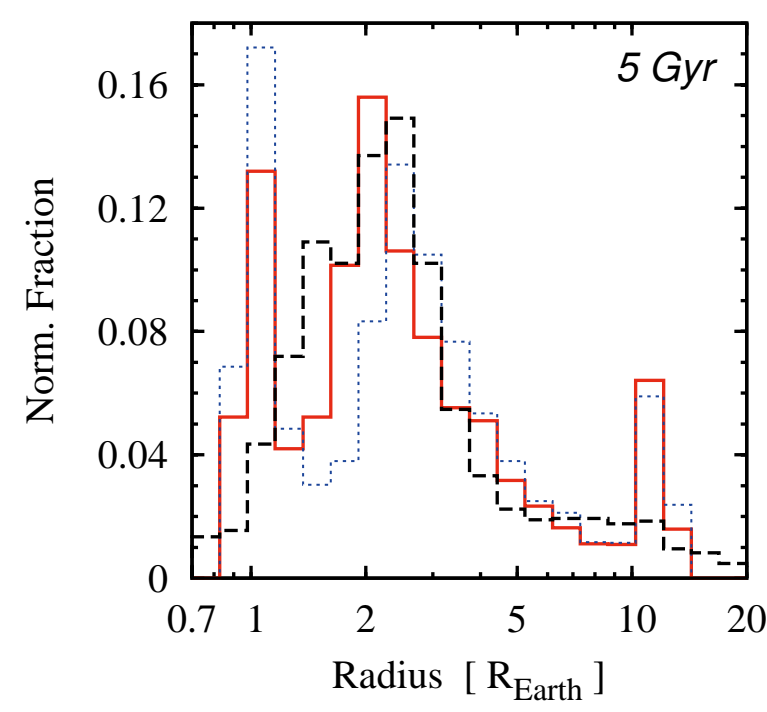

FIG. 16. - The size distributions of the planet within $1 \mathrm{AU}$ in the XE and GREY simulations at 5 Gyr. The blue dotted line shows the XE simulation. The red solid line shows the GREY simulation. The black dashed line shows the size distribution of all Kepler candidates.

tion are included). Figure 15 compares the $a-R$ distributions of the GREY and XE simulations. The planetary radii are smaller for the GREY model, as expected (Guillot 2010), but the global effects of evaporation in general and for the evaporation valley in particular remain visible in a similar manner in the $a-R$ space. The GREY simulation produces a narrower gap in the radius distribution because the planetary envelopes from this numerical experiment are less bloated than in simulations using the semi-grey model. Figure 16 compares the planet size distributions from the GREY and XE simulations at 5 Gyr. The GREY simulation clearly shows a bimodal distribution at approximately $2 R_{\oplus}$. The difference is that, compared with XE, the peak of the bare, rocky cores in the GREY simulation is less prominent, and the outer peak in the bimodal distribution occurs at a smaller size.

\subsection{Simplifications in the Evaporation Models}

An accurate method to calculate the mass-loss rate due to hydrodynamic escape is to solve the mass, energy, and momentum conservation equations (Tian et al. 2005; Penz et al. 2008; Murrav-Clav et al. 2009; Owen \& Jackson 2012; Lammer et al. 2013). In our work, we used an approximate mass-loss formula in the energy-limited and radiation-recombination-limited regime as well as criteria for determining whether the outflow is EUV- or X-ray-driven; we derived these from different authors (Murray-Clav et al. 2009; Jackson et al. 2012; Owen \& Jackson 2012). Thus, our evaporation model includes significant simplifications. In reality, the evaporation efficiency that characterizes the how much heating energy is converted to $\operatorname{Pd} V$ work depends on the characteristics of a planet and changes with time (Yelle 2004; Tian et al. 2005; Owen \& Jackson 2012). Moreover, the direct transition from an X-ray regime to an EUV-driven regime, as used in our model, can produce a discontinuous change in the mass-loss rate, as shown in Figure 3. Due to these limitations, we performed several groups of population syntheses using different evaporation models, which allowed us to obtain a likely range of mass-loss rates. Our results show that the evolution and final structure of a single planet may be less accurate due to the evaporation model simplifications but that the statistical information for the entire planet population was not greatly influenced.

For example, the XE2 simulation yields results that are similar to those of the XE simulation even though the heating efficiencies in the XE2 simulation are twice those of XE. The final mass and radius distributions are not sensitive to heating efficiency for the following reasons. At a fixed incident flux, the evaporation threshold scales are $M_{\text {env }} M_{\mathrm{p}} /\left(\epsilon R_{\mathrm{p}}^{3}\right)$. As shown in the NoEV panel in Figure 10, the initial $M_{\text {env }}$ of a planet increases quickly with the planetary mass. For example, the initial $f_{\text {env/core }}$ of a $4 M_{\oplus}$ planet is typical of $\sim 10 \%$; however, for a $2 M_{\oplus}$ planet, $\sim 1 \%$ is typical. For $R_{\mathrm{p}}$, the NoEV panel in Figure 11 shows that the increase of the planetary radius with an increasing planetary mass is not significant for low-mass planets with $M_{\mathrm{p}}<10 M_{\oplus}$. Thus, planetary mass plays a dominant role in determining the evaporation threshold. Moreover, atmospheric evaporation is only intense in the early stage for a short time (with a timescale $\sim 100 \mathrm{Myr}$ ). When increasing the heating efficiency in an evaporation model, a slow increase is observed for the critical planetary mass below which a planet will be evaporated to a bare rocky core. As shown in Figure 14, the final planet size distributions from the XE and XE2 simulations only show limited differences. One exception is the B04 simulation, where even Jovian planets lose a significant portion of their envelopes; in this case, the bimodal feature at the small planet size disappears because many Neptunian or Jovian planets evaporate to bare, rocky cores. These massive cores finally fill the deficit at $\sim 2 R_{\oplus}$.

Another simplification in our models is that we apply hydrodynamic evaporation to all planets, even for those at large semi-major axes. In fact, the atmospheric escape of planets at large distances should be included in the Jeans escape regime. Whether an escape flow is in the hydrodynamic or Jeans regime can be assessed by comparing the mean free path of gas molecules with the local scale height of the flow (Johnson et al. (2013) demonstrate how to implement this criterion in the energylimited model). Owen \& Jackson (2012) show that, for close-in planets with semi-major axes smaller than 0.1 $\mathrm{AU}$, the dominant mass-loss process is hydrodynamic evaporation. They found that at very small distances, such as $<0.05 \mathrm{AU}$, even gas giants with a few Jupiter masses can lose mass hydrodynamically. They also show that massive planets with large densities are too gravitationally bound for hydrodynamic outflow. For example, a planet that is more massive than $1 M_{\text {Jupiter }}$ with a density greater than $1 \mathrm{~g} \mathrm{~cm}^{-3}$ can no longer undergo hydrodynamic evaporation at $\sim 1$ AU. Thus, our models overestimate the mass-loss rates of the planets at large distances by assuming that the planets undergo hydrodynamic evaporation. However, this assumption does not affect the main statistical results for the entire planet population because only low-mass planets with tenuous 
envelopes can be evaporated to bare cores at large distances due to the overestimated mass-loss rates. We also did not include non-thermal ion escape in our models (e.g., the planetary ions that are captured by stellar wind). The influence of non-thermal escape on the statistical results is also weak because the mass-loss rate of ion pick-up escape is typically several times smaller than for thermal atmospheric escape (Kislyakova et al.|2013).

\subsection{Dependence on Stellar Type}

Most Kepler candidates orbit a host star with a mass of $0.8-1.1 M_{\odot}$. During the formation and evolution of our synthetic populations, all planets orbit around a sunlike star with a mass of exactly $1 M_{\odot}$. The key effect of different stellar types is that they lead to different disk properties, which finally determine the characteristics of the synthetic planet populations (Ida \& Lin 2005; Alibert et al. 2011). Massive F/G-type stars are found to yield fewer Neptunian planets but more massive Jovian planets. K/M-dwarfs are predicted to yield more Neptunian planets but fewer Jovian planets (Ida \& Lin 2005; Alibert et al. 2011). However, for the low-mass planets, which are sensitive to evaporation and form a bimodal size distribution, the fraction does not strongly depend on the mass of the central star (Ida \& Lin 2005; Alibert et al. 2011). Thus, at least for 0.8-1.1 $M_{\odot}$, comparisons between Kepler candidates and our synthetic planet populations are feasible.

The stars in our simulations all have the same $L_{\mathrm{EUV}}$ and $L_{\mathrm{X}}$, which follows the temporal evolution of the Xrays and EUV emissions of a sun-like star. In reality, the EUV flux (360-920 ̊) of a sun-like star at different times does not have direct observational constraints, with the exception of the present Sun. The temporal evolution of the EUV flux adopted in this work is from Ribas et al. (2005), which was derived by scaling the EUV flux with the temporal evolution of the stellar flux in other wavelength ranges. The accuracy of this method is approximately 10\% - 20\% (Ribas et al. 2005). Considering that the EUV evaporation contributes typically less than $10 \%$ of the total mass-loss of a planet (Owen \& Wu 2013), the low accuracy of the EUV fluxes at different times does not affect the statistical results. On the other hand, the stellar X-ray emissions are highly diverse (Güdel 2004). The ratio of X-rays to bolometric luminosity during the early saturated phase decreases from $10^{-3.1}$ for late $\mathrm{K}$ dwarfs to $10^{-4.3}$ for early F-type stars $\left(0.29 \leq(B-V)_{0}<\right.$ 1.4) (Jackson et al. 2012). Thus, our model is too deterministic because we use a fixed evolution track of stellar X-ray emission. M dwarfs can have strong chromosphere activities. They are very bright regarding hard radiation, which can strongly erode the planetary envelope. However, planets around K/M-dwarfs also have a less effective Roche lobe effect (Lammer et al. 2009; Penz \& Micela 2008). For planets surrounding F-, G-, $\mathrm{K}$-, and M-type stars, Lammer et al. (2009) show that evaporation can only remove a limited portion of the initial gas giant envelopes, and only Neptunian and terrestrial planets are significantly affected by evaporation.

\section{SUMMARY}

We combine models of hydrodynamic atmospheric escape with planet population syntheses which include both planet formation and evolution. Our global planet formation model is constructed based on the core accretion paradigm. We find that atmospheric escape adds characteristic features to the radius distribution of the synthetic planetary populations. The most interesting imprints are an evaporation valley in the radiusdistance diagram and a bimodal planet size distribution (cf. Owen \& Wu 2013). These features are consequences of evaporation, but their properties are also related to the characteristics of the initial planet population, and thus the planet formation process.

In our synthetic populations, the initial envelope fraction of low-mass planets with sizes of less than $4 R_{\oplus}$ is normally $<10 \%$ (especially for planets at small distances). The initial mass fraction of planetary envelopes also scales with the planetary core mass and is typically $<5 \%$ for Earth-size planets with a $1 M_{\oplus}$ core. Due to their low gravity, such very low-mass planets are sensitive to evaporation and are evaporated to bare cores with radii of about $1 R_{\oplus}$ at sufficiently small orbital distances. However, planets with larger core masses also have larger envelopes, as typical for the core accretion paradigm. At the end of evolution, such more massive super-Earths or Neptunian planets retain at least a portion of their $\mathrm{H} / \mathrm{He}$ envelopes. They will have substantially larger radii because already $0.1 \%$ in mass of $\mathrm{H} / \mathrm{He}$ significantly increases the radius. The threshold core mass where a complete loss of the initial $\mathrm{H} / \mathrm{He}$ envelope occurs decreases with orbital distance.

As a result, an "evaporation valley" running diagonally downward in the orbital distance - planetary radius plane appears. It separates bare cores from planets retaining some primordial $\mathrm{H} / \mathrm{He}$. As the process of losing the last, e.g., $0.1 \%$ of $\mathrm{H} / \mathrm{He}$ occurs on a short timescale, the valley is sparsely populated with planets at any given moment at late times (e.g., 5 Gyrs). At this time, the evaporation valley runs diagonally downward from about $2 R_{\oplus}$ at 0.06 AU to $1 R_{\oplus}$ at $0.5 \mathrm{AU}$.

Corresponding to this valley, the one-dimensional radius distribution of close-in low-mass planets is bimodal, with a local maximum at about $1 R_{\oplus}$, a local minimum at about $1.5 R_{\oplus}$ and another maximum at $2-3 R_{\oplus}$. The lower maximum in the bimodal distribution corresponds to the bare cores of planets that have lost their entire initial $\mathrm{H} / \mathrm{He}$ envelope. The minimum corresponds to the "evaporation valley". The second maximum corresponds to low-mass planets that have kept some primordial $\mathrm{H} / \mathrm{He}$.

No such very prominent features (deep diagonal evaporation valley and strong depletion at about $1.5 R_{\oplus}$ in the radius histogram) can be seen in the Kepler data for $R_{\mathrm{p}}<2 R_{\oplus}$, even if a small local minimum might be present also in the observational data (Owen \& Wu 2013; Petigura et al. 2013a). This difference could be due to the following reasons:

-First, our evaporation model might be too deterministic (identical mean XUV flux as a function of time for all stars) and/or overestimate the impact of evaporation.

-Second, in our formation model, all low-mass planets start with a primordial $\mathrm{H} / \mathrm{He}$ envelope and reach their final mass during the presence of the gaseous disk. In reality, at least some terrestrial planets will reach their final mass only after the dissipation of the gaseous nebula through a series of giant impacts, as likely for the Earth itself. This population of "late" planets is not expected 
to start with a significant $\mathrm{H} / \mathrm{He}$ envelope (a few percent), and therefore will not exhibit the imprints of evaporation (no significant radius evolution in time). Such planets would fill in the valley. For such planets, the transition from solid planets to planets with $\mathrm{H} / \mathrm{He}$ should likely not be a clear function of the semi-major axis, in contrast to the case that the transition is due to evaporation as studied here. This is an important constraint for formation models.

-Third, the composition of the bare cores might be different than in the model here: in our synthetic populations, the sizes of the bare evaporated cores range between 1.0 and $1.2 R_{\oplus}$ because all of the low-mass planets within 1 AU have rocky cores with a zero ice fraction despite orbital migration included in the formation model: they only migrate within the iceline. If the migration of individual planets is more efficient than assumed here, or if more massive planets push lower-mass planets closer-in due to capture in mean motion resonance in multiple systems (neither included here), planets that have formed beyond the ice line may migrate to close-in orbits during the formation stage. Such planets will accrete high amounts of ice during formation and, hence, have a large, icy core. In a forthcoming paper, we use a synthetic planet population with icy planetary cores to show that the sizes of the bare icy cores will be significantly larger and that these bare icy cores can fill in the minimum at $\sim 1.5 R_{\oplus}$. Thus, if close-in planets have both rocky and icy (or mixed) cores, this can lead to an approximately flat radius distribution for $R_{\mathrm{p}}<2-3 R_{\oplus}$, as observed in the bias-corrected radius distribution of the Kepler sample (e.g., Fressin et al. 2013; Petigura et al. 2013a). Thus, a diversity in the core composition combined with the consequences of evaporation may provide an explanation for the observed radius plateau to 2-3 $R_{\text {ops }}$ and the decrease at larger radii. Clearly, the ice content of closein low-mass planet is another fundamental constraint for formation (migration) models.

The specific shape and location of the second maximum at $2-3 R_{\oplus}$ in the bimodal distribution (planets that have retained primordial $\mathrm{H} / \mathrm{He}$ ) is related to envelope evaporation. Stronger evaporation produces a lower outer maximum and moves the peak to smaller radii. Most of our evaporation models lead to a similar outer peak, which is approximately consistent with the size distribution of the Kepler candidates in the radius range of about $2-8 R_{\oplus}$. However, in two extreme cases, the NoEV simulation without any evaporation and the B04 simulation with very strong evaporation (100\% heating efficiency), the final planet size distribution shows clear differences compared with the Kepler data in this range. This indicates that evaporation is indeed important in shaping the characteristics of close-in, low-mass planets (Lopez et al. 2012; Owen \& Wu 2013). For the comparison of observations with predictions of formation model for such planets, it should therefore be taken into ac- count. Other major findings are as follows: We find that in contrast to the radius distribution, the mass distribution of the entire planet population is barely affected by evaporation at $a>0.06 \mathrm{AU}$ : low-mass planets may lose all $\mathrm{H} / \mathrm{He}$, but its initial mass fraction is low anyway. Giant planets in contrast do not lose much $\mathrm{H} / \mathrm{He}$ in our nominal evaporation model - their high mass gravity protects them.

We demonstrate the importance of the core mass using a parameter study that is similar to the study described in Lopez et al. (2012). We confirm the evaporation threshold in the $M_{\mathrm{p}}^{2} / R_{\mathrm{p}}^{3}$ versus distance ${ }^{2}$ plane (Jackson et al. 2012; Lopez et al. 2012; Owen \& Wu 2013). Furthermore, we find that this evaporation threshold is also apparent in the mass-radius relationship of close-in planets. For the simulations that include evaporation, the mass-radius relationship clearly contains a threshold, and the very low-mass, very lowdensity planets that are initially above the threshold will lose at least a portion of their envelopes until they are below this threshold. Finally, the impact of the grain opacity in the outer radiative zone of protoplanets during the formation stage on the mass-radius relationship at 5 Gyrs remains clear also with evaporation.

Our study shows that several important observational constrains can be inferred from the comparison of observational results and theoretical formation and evolution models that include atmospheric escape. This is of high interest in view of several future high-precision photometric missions like TESS (Ricker et al. 2010) or CHEOPS (Broeg et al. 2013). Our results in particular show a dynamical evolution of the planetary population in terms of the radii (or composition) in time. In principle, such a temporal evolution could be observed directly with PLATO 2.0 (Rauer et al. 2013) which determines the ages of the host stars. This would open a new perspective to understand the nature of close-in planets.

We thank Dr. Jonathan Fortney for the atmospheric structures used for the comparison with the semi-grey model. We also thank Dr. Helmut Lammer, Kai-Martin Dittkrist, and Gabriel-Dominique Marleau for helpful discussions. S. Jin acknowledges the financial support of the Chinese Academy of Sciences and the Max-PlanckGesellschaft. This work was also supported by the National Natural Science Foundation of China (Grants No. 11273068, 11473073), the Natural Science Foundation of Jiangsu Province (Grant No. BK20141509), the innovative and interdisciplinary program by CAS (Grant No. KJZD-EW-Z001), and the Foundation of Minor Planets of the Purple Mountain Observatory. J. H. Ji acknowledges the Strategic Priority Research Program The Emergence of Cosmological Structures of the Chinese Academy of Sciences (Grant No. XDB09000000). C. Mordasini thanks the Max-Planck-Gesellschaft for the Reimar-Lüst Fellowship. We thank the referee for comments that helped to improve the manuscript.

\section{REFERENCES}

Adams, E. R., Seager, S., \& Elkins-Tanton, L. 2008, ApJ, 673, 1160

Alibert, Y., Carron, F., Fortier, A., et al. 2013, A\&A, 558, A109

Alibert, Y., Mordasini, C., \& Benz, W. 2011, A\&A, 526, A63
Alibert, Y., Mordasini, C., Benz, W., \& Winisdoerffer, C. 2005, A\&A, 434, 343

Baraffe, I., Selsis, F., Chabrier, G., et al. 2004, A\&A, 419, L13 
Batygin, K., Stevenson, D. J., \& Bodenheimer, P. H. 2011, ApJ, 738, 1

Beaugé, C., \& Nesvorný, D. 2013, ApJ, 763, 12

Bell, K. R., \& Lin, D. N. C. 1994, ApJ, 427, 987

Benz, W., Ida, S., Alibert, Y., Lin, D. N. C., \& Mordasini, C. 2014, arXiv:1402.7086 PPVI

Borucki, W. J., Koch, D. G., Basri, G., et al. 2011, ApJ, 728, 117

Broeg, C., Fortier, A., Ehrenreich, D., et al. 2013, European Physical Journal Web of Conferences, 47, 3005

Burrows, A., Marley, M., Hubbard, W. B., et al. 1997, ApJ, 491, 856

Dalgarno, A., \& McCray, R. A. 1972, ARA\&A, 10, 375

Dittkrist, K.-M., Mordasini, C., Klahr, H., Alibert, Y., \& Henning, T. 2014, A\&A, 567, A121

Dong, S., \& Zhu, Z. 2013, ApJ, 778, 53

Erkaev, N. V., Kulikov, Y. N., Lammer, H., et al. 2007, A\&A, 472,329

Fabrycky, D., \& Tremaine, S. 2007, ApJ, 669, 1298

Fortney, J. J., Lodders, K., Marley, M. S., \& Freedman, R. S. 2008, ApJ, 678, 1419

Fortney, J. J., Marley, M. S., Lodders, K., Saumon, D., \& Freedman, R. 2005, ApJ, 627, L69

Fouchet, L., Alibert, Y., Mordasini, C., \& Benz, W. 2012, A\&A, 540, A 107

Freedman, R. S., Marley, M. S., \& Lodders, K. 2008, ApJS, 174, 504

Fressin, F., Torres, G., Charbonneau, D., et al. 2013, ApJ, 766, 81

Goldreich, P., \& Tremaine, S. 1980, ApJ, 241, 425

Güdel, M. 2004, A\&A Rev., 12, 71

Guillot, T. 2010, A\&A, 520, A27

Guillot, T., \& Showman, A. P. 2002, A\&A, 385, 156

Hansen, B. M. S. 2008, ApJS, 179, 484

Hjellming, M. S., \& Webbink, R. F. 1987, ApJ, 318, 794

Howard, A. W., Marcy, G. W., Bryson, S. T., et al. 2012, ApJS, 201,15

Ida, S., \& Lin, D. N. C. 2004, ApJ, 604, 388

-. 2005, ApJ, 626, 1045

Ikoma, M., \& Hori, Y. 2012, ApJ, 753, 66

Jackson, A. P., Davis, T. A., \& Wheatley, P. J. 2012, MNRAS, 422,2024

Ji, J., Jin, S., \& Tinney, C. G. 2011, ApJ, 727, L5

Johnson, R. E., Volkov, A. N., \& Erwin, J. T. 2013, ApJ, 768, L4

Johnstone, D., Hollenbach, D., \& Bally, J. 1998, ApJ, 499, 758

Kislyakova, K. G., Lammer, H., Holmström, M., et al. 2013, Astrobiology, 13, 1030

Kley, W., Bitsch, B., \& Klahr, H. 2009, A\&A, 506, 971

Kurokawa, H., \& Nakamoto, T. 2014, ApJ, 783, 54

Lammer, H., Erkaev, N. V., Odert, P., et al. 2013, MNRAS, 430, 1247

Lammer, H., Selsis, F., Ribas, I., et al. 2003, ApJ, 598, L121

Lammer, H., Odert, P., Leitzinger, M., et al. 2009, A\&A, 506, 399

Lammer, H., Stökl, A., Erkaev, N. V., et al. 2014, MNRAS, 439, 3225

Lin, D. N. C., Bodenheimer, P., \& Richardson, D. C. 1996 , Nature, 380, 606

Lopez, E. D., \& Fortney, J. J. 2013, ApJ, 776, 2

Lopez, E. D., Fortney, J. J., \& Miller, N. 2012, ApJ, 761, 59

Marley, M. S., \& McKay, C. P. 1999, Icarus, 138, 268

Marley, M. S., Saumon, D., Guillot, T., et al. 1996, Science, 272, 1919

Marley, M. S., Seager, S., Saumon, D., et al. 2002, ApJ, 568, 335

Masset, F. S., D'Angelo, G., \& Kley, W. 2006, ApJ, 652, 730

Mayor, M., Marmier, M., Lovis, C., et al. 2011, arXiv:1109.2497

McKay, C. P., Pollack, J. B., \& Courtin, R. 1989, Icarus, 80, 23
Mordasini, C., Alibert, Y., \& Benz, W. 2009, A\&A, 501, 1139

Mordasini, C., Alibert, Y., Klahr, H., \& Henning, T. 2012a, A\&A, 547, A111

Mordasini, C., Alibert, Y., Georgy, C., et al. 2012b, A\&A, 547, A112

Mordasini, C., Klahr, H., Alibert, Y., Benz, W., \& Dittkrist, K.-M. 2010, arXiv:1012.5281

Mordasini, C., Klahr, H., Alibert, Y., Miller, N., \& Henning, T. 2014a, A\&A, 566, A141

Mordasini, C., Mollière, P., Dittkrist, K.-M., Jin, S., \& Alibert, Y. 2014b, arXiv:1406.5604, IJA Accepted

Movshovitz, N., Bodenheimer, P., Podolak, M., \& Lissauer, J. J. 2010, Icarus, 209, 616

Murray-Clay, R. A., Chiang, E. I., \& Murray, N. 2009, ApJ, 693, 23

Ormel, C. W., Ida, S., \& Tanaka, H. 2012, ApJ, 758, 80

Owen, J. E., \& Jackson, A. P. 2012, MNRAS, 425, 2931

Owen, J. E., \& Wu, Y. 2013, ApJ, 775, 105

Paardekooper, S.-J., \& Mellema, G. 2008, A\&A, 478, 245

Parmentier, V., \& Guillot, T. 2014, A\&A, 562, A133

Parmentier, V., Showman, A. P., \& Lian, Y. 2013a, A\&A, 558, A91

Parmentier, V., Guillot, T., Fortney, J. J., \& Marley, M. S. 2013b, arXiv:1311.6322

Penz, T., \& Micela, G. 2008, A\&A, 479, 579

Penz, T., Erkaev, N. V., Kulikov, Y. N., et al. 2008,

Planet. Space Sci., 56, 1260

Petigura, E. A., Marcy, G. W., \& Howard, A. W. 2013a, ApJ, 770,69

Petigura, E. A., Howard, A. W., \& Marcy, G. W. 2013b,

Proceedings of the National Academy of Science, 110, 19273

Rauer, H., Catala, C., Aerts, C., et al. 2013, arXiv:1310.0696

Ribas, I., Guinan, E. F., Güdel, M., \& Audard, M. 2005, ApJ, 622,680

Ricker, G. R., Latham, D. W., Vanderspek, R. K., et al. 2010, Bulletin of the American Astronomical Society, 42, \#450.06

Rogers, L. A., Bodenheimer, P., Lissauer, J. J., \& Seager, S. 2011, ApJ, 738, 59

Saumon, D., Chabrier, G., \& van Horn, H. M. 1995, ApJS, 99, 713

Showman, A. P., Fortney, J. J., Lian, Y., et al. 2009, ApJ, 699, 564

Tanaka, H., Takeuchi, T., \& Ward, W. R. 2002, ApJ, 565, 1257

Terquem, C., \& Papaloizou, J. C. B. 2007, ApJ, 654, 1110

Tian, F., Toon, O. B., Pavlov, A. A., \& De Sterck, H. 2005, ApJ, 621,1049

Uribe, A. L., Klahr, H., Flock, M., \& Henning, T. 2011, ApJ, 736, 85

Vazan, A., Kovetz, A., Podolak, M., \& Helled, R. 2013, MNRAS, 434,3283

Vidal-Madjar, A., Lecavelier des Etangs, A., Désert, J.-M., et al. 2008, ApJ, 676, L57

Vidal-Madjar, A., Désert, J.-M., Lecavelier des Etangs, A., et al. 2004, ApJ, 604, L69

Vidal-Madjar, A., Huitson, C. M., Bourrier, V., et al. 2013, A\&A, 560, A54

Watson, A. J., Donahue, T. M., \& Walker, J. C. G. 1981, Icarus, 48,150

Weidenschilling, S. J., \& Marzari, F. 1996, Nature, 384, 619

Wu, Y., \& Lithwick, Y. 2013, ApJ, 772, 74

Wu, Y., \& Murray, N. 2003, ApJ, 589, 605

Yelle, R. V. 2004, Icarus, 170, 167

Zhou, J.-L., Aarseth, S. J., Lin, D. N. C., \& Nagasawa, M. 2005, ApJ, 631, L85 